\title{
30. INORGANIC GEOCHEMISTRY OF LEG 43 SEDIMENTS
}

\author{
I. O. Murdmaa, V. V. Gordeev, and E. M. Emelyanov, P. P. Shirshov Institute of Oceanology, USSR \\ Academy of Sciences, Moscow, USSR \\ and \\ E. S. Bazilevskaya, Geological Institute, USSR Academy of Sciences, Moscow, USSR.
}

\section{INTRODUCTION}

A representative variety of western North Atlantic deep-sea sediments and lithofacies was drilled during Leg 43, including a variety of clays, siliceous and calcareous oozes, and volcaniclastic deposits. Samples taken for chemical determinations embrace the main lithologic units and sediment types cored. They were analyzed in order to distinguish the geochemical behavior of elements during sedimentation, and especially how certain elements are concentrated or dispersed. Particular attention was paid to the possible enrichment of iron, manganese, and minor elements in some red or variegated clays, and comparison of these clays with "metalliferous" sediments. Modifications of the chemical composition of sediments resulting from the admixture of volcaniclastic materials, as well as diagenetic processes such as silicification, migration of $\mathrm{Fe}$ and $\mathrm{Mn}$ and their concentration under oxidizing or reducing conditions, were also investigated.

The methods of investigation included bulk X-ray fluorescence analysis. Phase analyses of $\mathrm{Fe}$ and $\mathrm{Mn}$ were carried out to determine the forms of these elements in the sediments. Routine wet chemical methods were used for $\mathrm{Fe}, \mathrm{Mn}$, and $\mathrm{Ti}$ determinations, and atomic adsorption for $\mathrm{Al}, \mathrm{Zn}, \mathrm{Ni}, \mathrm{Co}, \mathrm{Cr}$, and $\mathrm{Cu}$, and partly for $\mathrm{Fe}$ and $\mathrm{Mn}$.

The following phase analysis methods were used to determine different forms of $\mathrm{Fe}$ in sediments: soluble $\mathrm{Fe}^{+2}$ was detected by titration with bichromate after 5 minutes of boiling in $\mathrm{HCl}(5: 100)$. For total $\mathrm{Fe}^{+2}$ the same method was used after decomposition in a mixture of $\mathrm{HF}$ and $\mathrm{H}_{2} \mathrm{SO}_{4}$. $\mathrm{Fe}$ in $\mathrm{FeS}_{2}$ was determined in the insoluble residue after evaporation of the mixture of $\mathrm{HF}$ and $\mathrm{H}_{2} \mathrm{SO}_{4}$. Free oxides of $\mathrm{Fe}$ ("soluble $\mathrm{Fe}^{+3}$ ") were determined photolitically in mercury light. The Tamm oxalate reagent with $p \mathrm{H}=3.25$ (consisting of $12.61 \mathrm{~g}$ of oxalic acid and $24.9 \mathrm{~g}$ of ammonium oxalate per liter of water) was used. We assume that amorphous and poorly crystalline hydrated forms of $\mathrm{Fe}_{2} \mathrm{O}_{3}$ have been dissolved by the reagent, including dispersed hydrohematite and hydrogoethite, whereas crystalline goethite, hematite, and magnetite, as well as $\mathrm{Fe}$ silicates have not been affected.

All determinations were made on dried powdered samples without the removal of seawater salts.

\section{RESULTS}

Bulk composition (Table 1) was determined in the non-calcareous or sparsely calcareous sediments of all main lithologic units. The most important constituent of the sediments is clay; that is, fine-grained (less than $4 \mu \mathrm{m}$ ) silicate matrix, composed of clay minerals with more or less abundant, fine-grained, clastic minerals (quartz, feldspar), amorphous or crystallized iron hydroxides, and other still unknown amorphous matter. The chemical composition of terrigenous clay in the investigated region may most likely be inferred from the analyses of Miocene hemipelagic clay drilled at Sites 382 and 385 (Table 1, Sections 382-7-2 and 385-3-2). Taking these quite similar analyses as representing "normal" terrigenous clay, it is possible to trace characteristic deviations in bulk analyses of other lithologic units. The deviations result from mixing of the clay matrix with various components, such as terrigenous sand and silt, volcaniclastic material, biogenic silica, and carbonate. However, changes in chemical composition of clayey sediments may also result from diagenetic alteration (silicification, zeolitization) since other types of clay, different from that taken as "normal," may occur.

The two samples from the upper terrigenous turbidites of Site 382 (1-5, 3-1, Table 1) are slightly calcareous; $\mathrm{CaO}$ and the fraction lost on ignition (i.1.) are, therefore higher, other compounds being correspondingly lower, except for $\mathrm{MgO}$ and $\mathrm{K}_{2} \mathrm{O}$. The last reflect essentially a higher content of mica in the clay mineral fraction, as determined by X-ray diffraction (see Koch and Rothe, this volume). The $\mathrm{SiO}_{2} / \mathrm{Al}_{2} \mathrm{O}_{3}$ ratio of both samples is, however, similar to that of the "normal" clays ( 3.3 to 3.5 ).

Eocene siliceous (radiolarian) mud and ooze of Subunit 4A at Site 386 (Sample 14-4, 15-21 cm), as well as at Site 387 (Samples 7-3, 80-83 cm; 10-5, 89$91 \mathrm{~cm}$ ), show higher $\mathrm{SiO}_{2}$ with respect to $\mathrm{Al}_{2} \mathrm{O}_{3}$ and other components. Extremely high concentrations of silica occur in lithified varieties of the Eocene siliceous turbidites. At Site 386 (Cores 27 to 34, Table 1) $\mathrm{SiO}_{2}$ ranges from 46.7 to 83.5 per cent. In many cases it is masked by $\mathrm{CaCO}_{3}$. The $\mathrm{SiO}_{2} / \mathrm{Al}_{2} \mathrm{O}_{3}$ ratio in these samples ranges from 4.1 to 14.6, showing essential enrichment in silica, as compared with the the clay matrix. At Site 387 (Cores 18 to 26, Table 1), $\mathrm{SiO}_{2}$ content ranges from 57.1 to 80.9 per cent, and $\mathrm{SiO}_{2} / \mathrm{Al}_{2} \mathrm{O}_{3}$ from 5.8 to 25.2 per cent. Extensive silicification is apparent in these rocks, which in some cases do not contain any visible biogenic siliceous particles.

Relatively high $\mathrm{SiO}_{2}$ contents (60.8 to $71.8 \%$ ) and $\mathrm{SiO}_{2} / \mathrm{Al}_{2} \mathrm{O}_{3}$ ratios (4.5 to 7.8) were determined for the red and variegated claystones of Site 386 (Cores 38 to 
TABLE 1

Bulk Chemical Composition of Leg 43 Sediments (x-ray fluorescence analysis)

\begin{tabular}{|c|c|c|c|c|c|c|c|c|c|c|c|c|}
\hline $\begin{array}{c}\text { Sample } \\
\text { (Interval in } \mathrm{cm} \text { ) }\end{array}$ & $\mathrm{SiO}_{2}$ & $\mathrm{TiO}_{2}$ & $\mathrm{Al}_{2} \mathrm{O}_{3}$ & $\mathrm{Fe}_{2} \mathrm{O}_{3}^{\mathrm{a}}$ & $\mathrm{MnO}$ & $\mathrm{MgO}$ & $\mathrm{CaO}$ & $\mathrm{K}_{2} \mathrm{O}$ & $\mathrm{Na}_{2} \mathrm{O}$ & $\mathrm{P}_{2} \mathrm{O}_{5}$ & i.l. & $\begin{array}{c}\mathrm{SiO}_{2} \\
\mathrm{Al}_{2} \mathrm{O}_{3}\end{array}$ \\
\hline \multicolumn{13}{|l|}{ Site 382} \\
\hline $\begin{array}{l}1-5,110-112 \\
3-1,126-128 \\
7-2,81-83 \\
15-6,60-62 \\
16-6,45-48 \\
20-2,36-38 \\
20-5,55-57 \\
21-3,82-84 \\
25-2,49-51\end{array}$ & $\begin{array}{l}45.8 \\
50.0 \\
57.8 \\
55.1 \\
36.2 \\
39.5 \\
39.1 \\
41.7 \\
32.4\end{array}$ & $\begin{array}{l}0.57 \\
0.68 \\
0.84 \\
0.65 \\
6.17 \\
4.60 \\
2.96 \\
4.83 \\
4.16\end{array}$ & $\begin{array}{l}12.9 \\
15.2 \\
17.0 \\
5.69 \\
12.4 \\
14.9 \\
12.1 \\
14.4 \\
11.1\end{array}$ & $\begin{array}{r}4.64 \\
6.58 \\
7.43 \\
5.97 \\
17.4 \\
11.8 \\
12.7 \\
10.6 \\
12.3\end{array}$ & $\begin{array}{l}0.08 \\
0.07 \\
0.05 \\
1.38 \\
0.40 \\
0.24 \\
0.11 \\
0.15 \\
0.20\end{array}$ & $\begin{array}{l}5.85 \\
3.88 \\
2.49 \\
3.87 \\
1.90 \\
4.28 \\
9.12 \\
5.20 \\
2.7\end{array}$ & $\begin{array}{l}9.77 \\
6.29 \\
0.48 \\
0.67 \\
3.03 \\
3.09 \\
4.51 \\
2.40 \\
12.2\end{array}$ & $\begin{array}{l}2.96 \\
3.32 \\
2.93 \\
3.23 \\
2.63 \\
3.95 \\
3.05 \\
5.23 \\
3.72\end{array}$ & $\begin{array}{l}\text { n.d.c } \\
\text { n.d. } \\
1.47 \\
1.56 \\
2.79 \\
3.26 \\
2.63 \\
5.56 \\
1.31\end{array}$ & $\begin{array}{l}\text { n.d. } \\
\text { n.d. } \\
0.12 \\
0.35 \\
0.04 \\
0.21 \\
1.15 \\
0.66 \\
0.18\end{array}$ & $\begin{array}{r}14.4 \\
12.2 \\
9.4 \\
11.8 \\
15.7 \\
13.8 \\
12.3 \\
9.7 \\
19.5\end{array}$ & $\begin{array}{l}3.5 \\
3.3 \\
3.4 \\
3.5 \\
2.9 \\
2.6 \\
3.2 \\
2.9 \\
2.9\end{array}$ \\
\hline \multicolumn{13}{|l|}{ Site 385} \\
\hline $\begin{array}{l}3-2,130-132 \\
8-5,130-132 \\
10-1,138-140 \\
11-2,24-26 \\
13-3,109-113 \\
14-1,66-70 \\
14-2,6-8 \\
15-1,53-55 \\
16-2,8-10 \\
16-2,123-124 \\
16-3,77-80 \\
16-2,122-123 \\
23-1,128-134 \\
23-3,142-150\end{array}$ & $\begin{array}{l}57.0 \\
57.1 \\
69.6 \\
53.0 \\
33.7 \\
53.3 \\
52.9 \\
51.1 \\
48.7 \\
48.2 \\
48.4 \\
50.2 \\
38.8 \\
18.4\end{array}$ & $\begin{array}{l}0.84 \\
0.58 \\
0.57 \\
0.61 \\
0.48 \\
0.87 \\
0.79 \\
1.00 \\
3.46 \\
2.15 \\
1.08 \\
2.69 \\
3.86 \\
4.56\end{array}$ & $\begin{array}{l}16.1 \\
15.2 \\
10.0 \\
14.5 \\
12.7 \\
19.3 \\
18.6 \\
19.4 \\
14.8 \\
15.76 \\
19.1 \\
15.6 \\
11.5 \\
6.90\end{array}$ & $\begin{array}{r}6.40 \\
5.75 \\
4.26 \\
6.72 \\
7.15 \\
8.18 \\
7.02 \\
9.87 \\
11.2 \\
11.78 \\
8.78 \\
10.4 \\
15.2 \\
15.6\end{array}$ & $\begin{array}{l}0.06 \\
0.09 \\
0.15 \\
0.75 \\
0.54 \\
0.40 \\
0.22 \\
0.15 \\
0.29 \\
0.68 \\
1.66 \\
0.01 \\
0.15 \\
0.17\end{array}$ & $\begin{array}{l}3.22 \\
2.94 \\
1.92 \\
3.18 \\
2.23 \\
2.39 \\
2.85 \\
2.29 \\
3.83 \\
3.19 \\
2.45 \\
2.68 \\
2.98 \\
4.15\end{array}$ & $\begin{array}{c}0.31 \\
0.49 \\
0.47 \\
0.92 \\
18.6 \\
0.33 \\
0.41 \\
0.38 \\
1.00 \\
1.17 \\
0.74 \\
1.41 \\
10.5 \\
18.3\end{array}$ & $\begin{array}{l}2.18 \\
3.02 \\
1.70 \\
3.32 \\
1.48 \\
2.98 \\
3.30 \\
3.11 \\
3.64 \\
4.87 \\
4.65 \\
4.97 \\
5.90 \\
2.0\end{array}$ & $\begin{array}{l}2.40 \\
2.07 \\
\text { n.d. } \\
\text { n.d. } \\
1.90 \\
1.05 \\
1.70 \\
1.23 \\
1.52 \\
\text { n.d. } \\
1.06 \\
\text { n.d. } \\
1.88 \\
4.15\end{array}$ & $\begin{array}{l}0.09 \\
0.19 \\
\text { n.d. } \\
\text { n.d. } \\
0.14 \\
0.15 \\
3.13 \\
0.15 \\
0.50 \\
\text { n.d. } \\
0.45 \\
\text { n.d. } \\
\text { n.d. } \\
0.41\end{array}$ & $\begin{array}{r}11.8 \\
11.4 \\
9.6 \\
13.8 \\
21.0 \\
11.1 \\
12.2 \\
11.1 \\
11.4 \\
11.4 \\
10.8 \\
10.6 \\
8.7 \\
25.3\end{array}$ & $\begin{array}{l}3.5 \\
3.8 \\
6.9 \\
3.7 \\
2.6 \\
2.8 \\
2.8 \\
2.6 \\
3.2 \\
3.0 \\
2.5 \\
3.2 \\
3.4 \\
2.7\end{array}$ \\
\hline
\end{tabular}

Site 386

\begin{tabular}{|c|c|c|c|c|c|c|c|c|c|c|c|c|}
\hline $4-2,111-115$ & 51.2 & 1.84 & 14.8 & 8.28 & 0.10 & 3.66 & 1.99 & 2.83 & n.d. & n.d. & 12.1 & 3.5 \\
\hline $6-4,128-133$ & 36.0 & 4.10 & 10.4 & 13.8 & 0.34 & 9.77 & 12.6 & 1.38 & 0.95 & 0.44 & 9.1 & 3.5 \\
\hline $8-1,147-150$ & 33.4 & 3.84 & 11.3 & 10.5 & 3.00 & 7.05 & 11.0 & 2.19 & 0.90 & 0.44 & 16.7 & 3.0 \\
\hline $8-6,134-137$ & 32.8 & 4.62 & 9.70 & 12.3 & 4.06 & 9.48 & 14.8 & 1.34 & 1.51 & 0.34 & 9.6 & 3.4 \\
\hline $9-3,127-130$ & 34.3 & 4.37 & 10.4 & 12.6 & 0.27 & 9.38 & 14.3 & 1.99 & 1.53 & 0.55 & 10.4 & 3.3 \\
\hline $13-3,127-133$ & 32.2 & 2.03 & 5.75 & 6.25 & 0.25 & 3.50 & 23.2 & 1.99 & 1.21 & 0.43 & 23.0 & 5.6 \\
\hline $14-4,19-21$ & 54.2 & 0.59 & 12.5 & 7.76 & 0.05 & 3.35 & 2.17 & 2.19 & 1.68 & 0.19 & 14.8 & 4.3 \\
\hline $28-2,123-125$ & 57.2 & 0.39 & 9.52 & 4.71 & 0.09 & 2.50 & 7.91 & 1.29 & 1.38 & 0.05 & 15.0 & 6.0 \\
\hline $28-4,108-110$ & 65.5 & 0.34 & 8.10 & 3.18 & 0.03 & 2.20 & 5.75 & 1.22 & 0.76 & 0.14 & 12.6 & 8.1 \\
\hline $30-2,89-91$ & 83.5 & 0.23 & 5.70 & 1.16 & 0.01 & 0.49 & 0.64 & 0.89 & 1.15 & n.d. & 5.81 & 14.6 \\
\hline $30-6,59-61$ & 51.7 & 0.21 & 7.57 & 1.91 & 0.14 & 1.76 & 15.1 & 1.04 & 1.68 & 0.05 & 18.9 & 6.8 \\
\hline $31-1,6-9$ & 46.7 & 0.34 & 10.6 & 4.25 & 0.10 & 2.35 & 15.5 & 1.56 & 0.55 & 0.17 & 18.2 & 4.4 \\
\hline $34-6,29-31$ & 58.8 & 0.60 & 14.3 & 6.77 & 0.33 & 2.38 & 1.35 & 2.36 & n.d. & n.d. & 11.7 & 4.1 \\
\hline $36-5,74-76$ & 52.8 & 0.87 & 19.1 & 7.46 & 0.72 & 2.33 & 0.74 & 3.33 & 0.90 & 0.23 & 11.4 & 2.8 \\
\hline $36-5,94-98$ & 52.7 & 0.83 & 19.4 & 7.39 & 0.66 & 3.42 & 0.68 & 2.09 & 0.20 & 0.17 & 11.9 & 2.7 \\
\hline $37-3,8-9$ & 64.9 & 0.45 & 10.43 & 5.17 & 0.03 & 1.81 & 0.81 & 1.69 & n.d. & n.d. & 13.1 & 6.2 \\
\hline $37-3,15-17$ & 71.1 & 0.50 & 9.67 & 5.04 & 0.03 & 1.86 & 0.51 & 1.92 & n.d. & n.d. & 7.0 & 7.4 \\
\hline $38-1,31-32$ & 69.6 & 0.39 & 8.93 & 5.08 & 1.46 & 1.33 & 0.58 & 1.22 & 2.54 & n.d. & 8.88 & 7.8 \\
\hline $38-3,90-91$ & 68.3 & 0.50 & 10.5 & 5.95 & 0.27 & 1.48 & 0.82 & 1.46 & 1.37 & 0.22 & 9.2 & 6.5 \\
\hline $39-1,129-131$ & 63.8 & 0.56 & 13.2 & 7.00 & 0.11 & 1.33 & 0.81 & 1.82 & 1.20 & 0.22 & 9.6 & 4.8 \\
\hline $39-1,144-145$ & 60.8 & 0.53 & 13.4 & 7.23 & 1.05 & 2.08 & 0.74 & 1.68 & 1.49 & 0.26 & 10.4 & 4.5 \\
\hline $40-2,0-1$ & 71.8 & 0.38 & 9.54 & 3.34 & 0.23 & 1.32 & 0.64 & 1.41 & 1.66 & 0.21 & 8.9 & 7.5 \\
\hline $40-2,3-4$ & 63.4 & 0.51 & 12.7 & 8.55 & 0.52 & 1.38 & 0.68 & 1.72 & 0.01 & 0.21 & 10.4 & 5.0 \\
\hline $41-4,68-70$ & 64.3 & 0.45 & 11.43 & 8.57 & 0.06 & 1.47 & 0.71 & 1.69 & n.d. & n.d. & 9.3 & 5.6 \\
\hline $42-2,105-107$ & 83.6 & 0.09 & 3.17 & 1.22 & 0.54 & 1.12 & 1.86 & 0.27 & n.d. & 0.15 & 7.1 & 26.5 \\
\hline $44-3,20-21$ & 72.5 & 0.26 & 8.22 & 3.63 & 0.40 & 1.71 & 0.62 & 1.22 & n.d. & n.d. & 9.9 & 8.8 \\
\hline $44-3,23-25$ & 83.2 & 0.07 & 3.06 & 1.35 & 0.16 & 0.33 & 3.37 & 0.29 & n.d. & n.d. & 7.7 & 27.2 \\
\hline $44-3,23,5-25$ & 61.8 & 0.27 & 7.40 & 3.07 & 0.21 & 1.74 & 1.30 & 1.01 & n.d. & n.d. & 22.1 & 8.3 \\
\hline $48-3,123-124$ & 26.3 & 0.08 & 4.70 & 1.17 & 0.86 & 0.78 & 33.0 & 0.11 & n.d. & 0.10 & 32.4 & 5.3 \\
\hline $49-3,90-93$ & 63.4 & 0.34 & 8.68 & 4.52 & 1.94 & 2.26 & 5.09 & 1.25 & 0.92 & 0.06 & 11.4 & 7.3 \\
\hline $60-5,140-141$ & 64.5 & 0.41 & 9.50 & 5.05 & 0.26 & 2.48 & 1.86 & 1.90 & 1.02 & 0.18 & 12.4 & 6.8 \\
\hline $63-4,82-84$ & 57.1 & 0.38 & 8.44 & 13.55 & 0.04 & 1.43 & 0.43 & 3.28 & n.d. & n.d. & 15.6 & 6.8 \\
\hline $64-3,22-23$ & 71.3 & 0.36 & 7.02 & 7.03 & 0.06 & 2.35 & 0.37 & 2.69 & 0.87 & 0.10 & 5.3 & 10.2 \\
\hline $64-3,24-25$ & 70.7 & 0.33 & 7.32 & 10.2 & 0.05 & 2.28 & 0.30 & 2.53 & 0.79 & 0.17 & 5.4 & 9.7 \\
\hline $65-3,99-101$ & 51.9 & 0.39 & 7.94 & 16.04 & 0.03 & 3.17 & 0.73 & 2.24 & n.d. & n.d. & 16.9 & 6.5 \\
\hline \multicolumn{13}{|l|}{ Site 387} \\
\hline $7-3,80-83$ & 64.8 & 0.45 & 10.27 & 4.73 & 0.72 & 2.55 & 0.74 & 1.35 & n.d. & n.d. & 12.6 & 6.3 \\
\hline $10-5,89-91$ & 69.5 & 0.20 & 5.09 & 1.81 & 0.34 & 0.57 & 6.00 & 0.61 & n.d. & n.d. & 13.6 & 13.6 \\
\hline $18-1,81-83$ & 53.3 & 0.16 & 5.59 & 1.59 & 0.19 & 2.67 & 15.86 & 0.67 & n.d. & n.d. & 19.9 & 9.5 \\
\hline $18-1,81-83$ & 80.7 & 0.24 & 6.58 & 1.97 & 0.03 & 0.95 & 0.65 & 0.64 & n.d. & n.d. & 7.0 & 12.3 \\
\hline $18-1,84-86$ & 80.9 & 0.19 & 5.88 & 1.71 & 0.02 & 0.65 & 0.48 & 0.55 & n.d. & n.d. & 6.9 & 13.8 \\
\hline $19-1,103-108$ & 57.1 & 0.24 & 7.53 & 2.22 & 0.08 & 1.85 & 11.71 & 0.88 & n.d. & n.d. & 17.6 & 7.6 \\
\hline $19-2,61-63$ & 78.3 & 0.15 & 3.10 & 1.06 & 0.04 & 0.60 & 8.12 & 0.36 & n.d. & n.d. & 8.2 & 25.2 \\
\hline $19-3,20-22$ & 72.7 & 0.33 & 8.42 & 3.73 & 0.29 & 1.41 & 0.71 & 1.09 & n.d. & n.d. & 9.5 & 8.6 \\
\hline $25-3,144-146$ & 80.7 & 0.23 & 6.14 & 2.01 & 0.02 & 1.84 & 1.10 & 0.83 & 0.55 & 0.21 & 6.6 & 13.1 \\
\hline $26-2,0-2$ & 66.8 & 0.77 & 11.6 & 5.36 & 0.04 & 2.62 & 0.44 & 1.87 & 1.52 & 0.18 & 8.9 & 5.8 \\
\hline $29-2,12-15$ & 53.6 & 0.94 & 19.3 & 8.65 & 0.11 & 2.37 & 0.46 & 3.55 & 1.83 & 0.20 & 9.8 & 2.8 \\
\hline $34-4,96-98$ & 74.8 & 0.36 & 9.03 & 3.59 & 0.02 & 1.09 & 0.58 & 1.12 & n.d. & n.d. & 6.4 & 8.3 \\
\hline $34-4,117-119$ & 71.3 & 0.36 & 8.78 & 3.97 & 0.02 & 1.22 & 1.19 & 1.10 & n.d. & n.d. & 10.0 & 8.1 \\
\hline $37-2,101-103$ & 72.0 & 0.44 & 9.70 & 3.64 & 0.02 & 4.60 & 0.47 & 1.65 & 1.17 & 0.23 & 6.3 & 7.4 \\
\hline $42-1,72-75$ & 19.2 & 0.15 & 4.50 & 2.75 & 0.03 & 2.95 & 34.44 & 1.41 & 0.42 & 0.28 & 33.7 & 4.3 \\
\hline $50-1,35-38$ & 48.7 & 1.03 & 15.7 & 7.48 & 0.06 & 9.80 & 10.1 & 0.09 & 1.25 & 0.13 & 4.8 & 3.1 \\
\hline
\end{tabular}

aTotal $\mathrm{Fe}$ is expressed as $\mathrm{Fe}_{2} \mathrm{O}_{3}$, including $\mathrm{FeO}$.

$\mathrm{b}$ Loss on ignition.

$c_{\text {n.d. }}$ - not determined. 
40, Table 1), except in the upper red claystone interval (Core 36), where $\mathrm{SiO}_{2}$ is rather low and $\mathrm{Al}_{2} \mathrm{O}_{3}$ is high. Even more enriched in silica are the black and greenish gray claystones of Sites 386 (Cores 42 to 64) and 387 (Cores 34 to 37 , Table 1). The $\mathrm{SiO}_{2} / \mathrm{Al}_{2} \mathrm{O}_{3}$ ratio here reaches 26.5. It should be noted, that no rock samples described as "chert" or "porcellanite" were included in the analyzed set.

Volcaniclastic and zeolitic deposits drilled at Sites 382 (Cores 15 to 25), 385 (Cores 14 to 23), and 386 (Cores 6 to 13) (Table 1), are variable in their bulk chemical composition, but have some characteristic features in common. These are relatively low $\mathrm{SiO}_{2}$ content and $\mathrm{SiO}_{2} / \mathrm{Al}_{2} \mathrm{O}_{3}$ ratio, and high $\mathrm{Fe}_{2} \mathrm{O}_{3}$ and $\mathrm{TiO}_{2}$. In addition, the presence of basaltic volcaniclastic matter is probably responsible for high $\mathrm{MgO}$ at Site 382 in Unit 3 (up to $9.12 \%$ ), and at Site 386 in Unit 3 (up to $9.77 \%$ ). $\mathrm{K}_{2} \mathrm{O}$ is higher in zeolite-bearing sediments and volcaniclastic breccias of Sites 382 and 385, whereas volcaniclastic sandstones of the Site 386 are relatively low in potassium.

The red claystones of Site 386, Core 36, and Site 387, Core 29, are similar, showing high $\mathrm{Al}_{2} \mathrm{O}_{3}$ and a low $\mathrm{SiO}_{2} / \mathrm{Al}_{2} \mathrm{O}_{3}$ ratio, as in the volcaniclastic intervals, but without any enrichment in $\mathrm{TiO}_{2}$. Similar features appear in the upper part of vitric claystone sequence (Cores 13 and 14) at Site 385.

\section{Iron}

The distribution of iron in Recent sediments, as well as its behavior during sedimentation and diagenesis have been discussed at length in numerous publications. Total Fe was determined in 229 of the Leg 43 sediment samples (Table 2 ). The set of analyzed samples includes almost all of the important varieties of sediments cored during Leg 43, except for the shallowwater limestones at Site 384 . Wide limits in concentration occur, from less than 0.5 per cent in pure calcareous oozes and limestone up to 22.9 per cent in sideritic interbeds of the Site 386 black and gray claystone sequence. The values less than 2 per cent were found almost exclusively in calcareous sediments, where $\mathrm{Fe}$ is diluted by biogenic carbonate.

Most of the terrigenous sediments-clay and silty clay-are characterized by iron content from 2 to 5 per cent. The Miocene hemipelagic clay at Site 382 contains 4.12 to 7.68 per cent Fe. At Site 385 the Fe content is quite uniform (4.11 to 4.33 per cent). In Pliocene-Pleistocene turbidites of Site $382 \mathrm{Fe}$ content ranges from 1.39 to 6.12 per cent, the lower values reflecting quartzose sand and silt. In Pleistocene pelagic clay at Site $387,5.44$ per cent $\mathrm{Fe}$ was determined. In Paleocene to Eocene siliceous turbidites at Sites 386 and 387 , as well as in radiolarian mud at Sites 385 and $386, \mathrm{Fe}$ content is low, most values falling in the range of 2 to 4.5 per cent. Dilution by both biogenic and authigenic silica is important here.

The lowermost units of mainly red-colored sediments at Sites 382 (Unit 3) and 385 (Unit 3), interpreted as volcanogenic, are essentially enriched in iron
TABLE 2

Iron, Manganese, and Titanium Content in Leg 43 Sediments (wt \%)

\begin{tabular}{|c|c|c|c|c|c|c|c|c|}
\hline Sample & & & M & & & $\mathrm{i}$ & $\mathrm{Fe} / \mathrm{Ti}$ & $\mathrm{Mn} / \mathrm{Ti}$ \\
\hline (Interval in $\mathrm{cm}$ ) & a & $\mathrm{b}$ & a & b & a & $\mathrm{b}$ & Ratio & Ratio \\
\hline Site 382 & & & & & & & & \\
\hline $1-1,59-61$ & 4.40 & 4.89 & 0.06 & 0.07 & 0.49 & 0.54 & 9.0 & 0.1 \\
\hline $2-2,60-62$ & 3.86 & $* \mathrm{c}$ & 0.09 & ${ }^{*}$ & 0.44 & $*$ & 8.8 & 0.2 \\
\hline $1-3,126-128$ & 2.22 & $*$ & 0.03 & $*$ & 0.31 & $*$ & 7.2 & 0.1 \\
\hline $1-4,90-92$ & 2.21 & $*$ & 0.05 & $*$ & 0.28 & ${ }^{*}$ & 7.9 & 0.2 \\
\hline $1-4,113-115$ & 1.39 & 1.71 & 0.05 & 0.06 & 0.25 & 0.31 & 5.5 & 0.2 \\
\hline $1-4,127-129$ & 4.64 & $*$ & 0.06 & $*$ & 0.49 & $*$ & 9.5 & 0.1 \\
\hline $1-5,110-112$ & 4.08 & * & 0.10 & * & 0.45 & * & 9.1 & 0.2 \\
\hline $1-6,40-42$ & 2.60 & $*$ & 0.06 & * & 0.33 & * & 7.9 & 0.2 \\
\hline $2-1,60-70$ & 2.50 & * & 0.05 & * & 0.39 & * & 6.4 & 0.1 \\
\hline $2-2,90-92$ & 2.59 & * & 0.02 & * & 0.20 & * & 12.9 & 0.1 \\
\hline $2-3,48-50$ & 4.34 & * & 0.06 & * & 0.44 & * & 9.9 & 0.1 \\
\hline $2-3,64-66$ & 5.43 & * & 0.07 & * & 0.48 & * & 11.3 & 0.1 \\
\hline $2-3,92-94$ & 2.30 & * & 0.04 & * & 0.37 & * & 6.2 & 0.1 \\
\hline $3-1,126-128$ & 5.22 & * & 0.06 & * & 0.46 & * & 11.3 & 0.1 \\
\hline $3-2,35-37$ & 2.56 & * & 0.07 & $*$ & 0.38 & * & 6.7 & 0.2 \\
\hline $3-2,100-102$ & 6.12 & * & 0.06 & $*$ & 0.52 & * & 11.8 & 0.1 \\
\hline $3-2,147-149$ & 2.76 & * & 0.05 & $*$ & 0.43 & * & 6.4 & 0.1 \\
\hline $4-1,80-82$ & 4.92 & * & 0.08 & * & 0.50 & * & 9.8 & 0.2 \\
\hline $5-1,96-98$ & 5.12 & * & 0.06 & * & 0.50 & * & 10.2 & 0.1 \\
\hline $5-2,95-97$ & 4.95 & * & 0.28 & * & 0.52 & * & 9.5 & 0.5 \\
\hline $5-3,100-102$ & 5.18 & * & 0.05 & * & 0.55 & * & 9.4 & 0.1 \\
\hline $6-1,53-55$ & 6.47 & $*$ & 1.64 & $*$ & 0.46 & * & 14.1 & 3.6 \\
\hline $6-1,148-150$ & 4.61 & $*$ & 0.04 & ${ }^{*}$ & 0.50 & * & 9.2 & 0.1 \\
\hline $7-2,81-83$ & 5.00 & * & 0.05 & ${ }^{*}$ & 0.50 & * & 10.0 & 0.1 \\
\hline $7-6,67-69$ & 7.68 & * & 1.13 & * & 0.42 & * & 18.3 & 2.7 \\
\hline $8-3,108-110$ & 4.97 & * & 0.03 & * & 0.56 & * & 8.9 & 0.1 \\
\hline $8-5,97-99$ & 5.62 & * & 0.04 & * & 0.48 & * & 11.7 & 0.1 \\
\hline $9-1,100-102$ & 4.46 & * & 0.03 & * & 0.49 & * & 9.1 & 0.1 \\
\hline $9-5,120-122$ & 4.80 & * & 0.02 & * & 0.48 & * & 10.0 & 0.1 \\
\hline $10-2,4-7$ & 5.72 & * & 0.02 & * & 0.58 & * & 9.9 & 0.1 \\
\hline $11-1,96-98$ & 5.40 & * & 0.02 & * & 0.46 & * & 11.7 & 0.1 \\
\hline $11-4,76-78$ & 4.69 & * & 0.01 & * & 0.46 & * & 10.2 & 0.1 \\
\hline $12-2,90-92$ & 4.12 & * & 0.05 & * & 0.44 & * & 9.4 & 0.1 \\
\hline $12-4,103-105$ & 4.62 & $*$ & 0.03 & * & 0.44 & * & 10.5 & 0.1 \\
\hline $13-4,34-36$ & 5.36 & * & 0.05 & * & 0.40 & * & 13.4 & 0.1 \\
\hline & 4.36 & * & 0.0 & * & 0.43 & * & 10.1 & 0.1 \\
\hline $14-1,36-38$ & 4.57 & * & 0.03 & * & 0.44 & * & 10.4 & $\begin{array}{l}.1 \\
0.1\end{array}$ \\
\hline $14-5,123-125$ & 4.69 & * & 0.02 & * & 0.44 & * & 10.6 & 0.1 \\
\hline $15-3,7-9$ & 4.80 & $*$ & 0.02 & * & 0.48 & * & 10.0 & 0.1 \\
\hline $15-3,147-150$ & 6.14 & * & 0.03 & * & 0.47 & * & 13.1 & 0.1 \\
\hline $15-4,147-150$ & 6.25 & * & 0.05 & * & 0.47 & * & 13.3 & 0.1 \\
\hline $15-5,10-12$ & 5.00 & * & 0.08 & $*$ & 0.45 & $*$ & 11.1 & 0.2 \\
\hline $15-5,33-37$ & 5.23 & * & 0.02 & * & 0.32 & $*$ & 16.3 & 0.1 \\
\hline $15-6,44-47$ & 4.43 & * & 0.15 & $*$ & 0.25 & $*$ & 17.7 & 0.6 \\
\hline $15-6,44-47$ & 4.16 & * & 0.54 & $*$ & 0.30 & * & 13.9 & 1.8 \\
\hline $15-6,60-62$ & 3.88 & * & 0.72 & * & 0.25 & * & 15.5 & 2.9 \\
\hline $16-1,0-3$ & 6.10 & & 0.08 & & 0.50 & & 12.2 & 0.2 \\
\hline $16-1,9-13$ & 7.26 & & 0.02 & & 2.38 & & 3.0 & 0.1 \\
\hline $16-1,94-98$ & 5.00 & 5.56 & 0.76 & 0.84 & 0.58 & 0.64 & 8.7 & 1.3 \\
\hline $16-1,100-107$ & 4.65 & & 0.09 & & 0.96 & & 4.8 & 0.1 \\
\hline $16-2,0-3$ & 6.25 & 6.87 & 0.10 & 0.11 & 1.39 & 1.53 & 4.5 & 0.1 \\
\hline $16-4,99-105$ & 8.53 & & 0.18 & & 2.30 & & 3.7 & 0.1 \\
\hline $16-6,45-48$ & 10.00 & & 0.27 & & 1.44 & & 6.9 & 0.2 \\
\hline $16-6,123-126$ & 8.59 & 9.21 & 0.22 & 0.24 & $\begin{array}{l}1.50 \\
-1.54\end{array}$ & 1.66 & 5.7 & 0.2 \\
\hline $17-1,102-104$ & 7.97 & & 0.19 & & 2.11 & & 3.8 & 0.1 \\
\hline $17-3,116-118$ & 9.27 & & 0.37 & & 2.02 & & 4.6 & 0.2 \\
\hline $17-4,115-119$ & 6.81 & * & 0.05 & * & 1.25 & * & 5.4 & 0.5 \\
\hline $17-5,25-27$ & 6.95 & & 0.15 & & 1.66 & & 4.2 & 0.1 \\
\hline $17-5,79-81$ & 6.21 & & 0.14 & & 1.44 & & 4.3 & 0.1 \\
\hline $18-1,61-65$ & 6.72 & 7.21 & 0.23 & 0.25 & 1.14 & 1.22 & 5.9 & 0.2 \\
\hline $18-2,4-9$ & 3.92 & 6.78 & 0.12 & 0.21 & 0.76 & 1.31 & 5.2 & 0.2 \\
\hline $18-2,109-112$ & 5.88 & 7.77 & 0.12 & 0.16 & 1.09 & 1.44 & 5.4 & 0.1 \\
\hline $18-4,9-12$ & 6.44 & 8.15 & 0.15 & 0.19 & 1.02 & 1.29 & 6.3 & 0.2 \\
\hline $18-4,144-147$ & 8.15 & & 0.08 & & 1.20 & & 6.8 & 0.1 \\
\hline $19-4,20-25$ & 7.49 & 8.28 & 0.15 & 0.16 & 1.13 & 1.25 & 6.6 & 0.1 \\
\hline $19-4,84-88$ & 8.78 & & 0.19 & & 2.11 & & 4.2 & 0.1 \\
\hline $20-2 ? 36-38$ & 8.32 & & 0.22 & & 1.13 & & 7.4 & 0.2 \\
\hline $20-4,36-41$ & 7.00 & * & 0.15 & * & 1.63 & & 4.3 & 0.1 \\
\hline $20-4,129-133$ & 8.92 & * & 0.25 & * & 1.44 & * & 6.2 & 0.2 \\
\hline $20-5,55-57$ & 2.24 & * & 0.19 & * & 0.89 & * & 2.5 & 0.2 \\
\hline $21-3,82-84$ & 7.68 & * & 0.23 & $*$ & 1.25 & * & 6.1 & 0.2 \\
\hline $22-2,86-89$ & 6.63 & * & 0.14 & $*$ & 1.71 & $*$ & 3.9 & 0.1 \\
\hline $22-2,120-122$ & 7.22 & * & 0.16 & * & 1.73 & * & 4.2 & 0.1 \\
\hline $22-3,148-150$ & 8.11 & $*$ & 0.16 & $*$ & 2.02 & * & 4.0 & 0.1 \\
\hline $23-2,30-35$ & 7.98 & $*$ & 0.09 & $*$ & 1.25 & $*$ & 6.4 & 0.1 \\
\hline $24-1,90-93$ & 6.37 & 9.23 & 0.17 & 0.25 & 1.09 & 1.58 & 5.8 & 0.2 \\
\hline $25-2,49-51$ & 8.61 & & 0.15 & & 2.50 & & $\begin{array}{l}3.0 \\
3.4\end{array}$ & 0.1 \\
\hline $25, \mathrm{CC}$ & 5.00 & 8.15 & 0.11 & 0.18 & 0.63 & 1.02 & 8.0 & 0.2 \\
\hline Site 384 & & & & & & & & \\
\hline $1-2,28-30$ & & & & & & & & \\
\hline & 1.48 & $3.15 \mathrm{~d}$ & $\begin{array}{l}0.03 \\
0.07\end{array}$ & $0.15 \mathrm{~d}$ & 0.12 & $0.26^{\mathrm{d}}$ & 12.3 & 0.6 \\
\hline $2-5,118$ & 1.78 & $3.17^{\mathrm{d}}$ & 0.06 & $0.12^{\mathrm{d}}$ & 0.16 & $0.33^{\mathrm{d}}$ & 11.1 & 0.4 \\
\hline & 1.84 & $4.28^{\mathrm{d}}$ & 0.06 & $0.14^{\mathrm{d}}$ & 0.18 & $0.42^{\mathrm{d}}$ & 10.2 & 0.3 \\
\hline & 1.62 & $3.45^{\mathrm{d}}$ & 0.10 & $0.21^{\mathrm{d}}$ & 0.13 & $0.28^{\mathrm{d}}$ & 12.5 & 0.8 \\
\hline $4-5,122-124$ & 1.46 & $3.84^{\mathrm{d}}$ & 0.19 & $0.50^{\mathrm{d}}$ & 0.12 & $0.32^{\mathrm{d}}$ & 12.2 & 1.6 \\
\hline $4-6,114-115$ & 1.66 & $4.37^{\mathrm{d}}$ & 2.78 & $7.32^{\mathrm{d}}$ & 0.13 & $0.34^{\mathrm{d}}$ & 12.8 & 21.4 \\
\hline
\end{tabular}


TABLE 2 - Continued

\begin{tabular}{|c|c|c|c|c|c|c|c|c|}
\hline \multirow{2}{*}{$\begin{array}{c}\text { Sample } \\
\text { (Interval in } \mathrm{cm} \text { ) }\end{array}$} & \multicolumn{2}{|c|}{$\mathrm{Fe}$} & \multicolumn{2}{|c|}{$\mathrm{Mn}$} & \multicolumn{2}{|c|}{$\mathrm{Ti}$} & \multirow{2}{*}{$\begin{array}{l}\mathrm{Fe} / \mathrm{Ti} \\
\text { Ratio }\end{array}$} & \multirow{2}{*}{$\begin{array}{l}\mathrm{Mn} / \mathrm{T} \\
\text { Ratio }\end{array}$} \\
\hline & $\mathrm{a}$ & $\mathrm{b}$ & a & b & a & $\mathrm{b}$ & & \\
\hline $5-1,100-102$ & 0.42 & $2.00^{\mathrm{d}}$ & 0.08 & $0.38^{d}$ & $\operatorname{tr}^{\mathrm{e}}$ & - & - & - \\
\hline $6-2,118-120$ & 0.82 & $2.83^{\mathrm{d}}$ & 0.17 & $0.59^{\mathrm{d}}$ & 0.05 & $0.17 \mathrm{~d}$ & 16.4 & 3.4 \\
\hline $7-3,16-18$ & 0.63 & $3.00^{d}$ & 0.10 & $0.48^{\mathrm{d}}$ & 0.05 & $0.24^{\mathrm{d}}$ & 12.6 & 2.00 \\
\hline $8-2,83-85$ & 0.83 & $3.46^{\mathrm{d}}$ & 0.15 & $0.62^{\mathrm{d}}$ & 0.08 & $0.33^{d}$ & 10.4 & 1.9 \\
\hline $9-3,37-39$ & 1.07 & $2.55^{\mathrm{d}}$ & 0.18 & $0.43^{\mathrm{d}}$ & 0.12 & $0.29 \mathrm{~d}$ & 8.9 & 1.5 \\
\hline $10-6,30-32$ & 1.04 & $3.35^{\mathrm{d}}$ & 0.16 & $0.52^{\mathrm{d}}$ & 0.08 & $0.26^{d}$ & 13.0 & 2.0 \\
\hline $11-2,82-84$ & 0.90 & $2.50^{\mathrm{d}}$ & 0.08 & $0.22^{\mathrm{d}}$ & 0.08 & $0.22^{\mathrm{d}}$ & 4.1 & 1.0 \\
\hline $11-4,97-99$ & 1.18 & $3.93^{d}$ & 0.12 & $0.40^{\mathrm{d}}$ & 0.13 & $0.43^{\mathrm{d}}$ & 9.1 & 0.9 \\
\hline $12-5,117-119$ & 0.90 & $2.50^{\mathrm{d}}$ & 0.12 & $0.33^{\mathrm{d}}$ & 0.08 & $0.22^{\mathrm{d}}$ & 11.2 & 1.5 \\
\hline $13-2,86-88$ & 0.70 & $4.12^{\mathrm{d}}$ & 0.10 & $0.59^{\mathrm{d}}$ & 0.08 & $0.47^{\mathrm{d}}$ & 8.8 & 1.2 \\
\hline $13-5,88-90$ & 0.37 & $3.36^{\mathrm{d}}$ & 0.10 & $0.91^{\mathrm{d}}$ & 0.04 & $0.36^{\mathrm{d}}$ & 9.2 & 2.5 \\
\hline $14-2,92-94$ & 0.38 & $4.22^{\mathrm{d}}$ & 0.06 & $0.67^{\mathrm{d}}$ & 0.07 & $0.78^{d}$ & 5.4 & 0.8 \\
\hline $15-2,15-17$ & 0.34 & $4.25^{\mathrm{d}}$ & 0.06 & $0.75^{\mathrm{d}}$ & 0.04 & $0.50^{\mathrm{d}}$ & 8.5 & 1.5 \\
\hline
\end{tabular}

Site 385

$\begin{array}{lcccccccc}1-4,30-32 & 4.11 & * & 0.03 & * & 0.44 & * & 9.3 & 0.1 \\ 2-5,60-62 & 433 & * & 0.04 & * & 0.42 & * & 10.3 & 0.1 \\ 3-2,130-132 & 4.43 & * & 0.10 & * & 0.40 & * & 11.07 & 0.2 \\ 3-5,120-126 & 4.20 & * & 0.05 & * & 0.44 & * & 9.5 & 0.1 \\ 4-2,50-52 & 2.23 & * & 0.06 & * & 0.23 & * & 9.7 & 0.3 \\ 54,100-102 & 2.31 & * & 0.23 & * & 0.14 & * & 16.5 & 1.6 \\ 8-5,130-132 & 3.60 & * & 0.19 & * & 0.25 & * & 14.40 & 0.8 \\ 9-1,103-105 & 3.01 & * & 0.20 & * & 0.25 & * & 12.0 & 0.8 \\ 10-1,130-132 & 2.18 & * & 0.09 & * & 0.20 & * & 10.9 & 0.4 \\ 10-1,138-140 & 2.98 & * & 0.12 & * & 0.22 & * & 13.5 & 0.5 \\ 11-2,24-26 & 4.80 & * & 0.58 & * & 0.37 & * & 13.0 & 1.6 \\ 11-2,46-48 & 4.16 & * & 0.42 & * & 0.35 & * & 11.9 & 1.2 \\ 12-2,15-17 & 2.39 & 3.88 \text { d } & 0.22 & 0.34 \text { d } & 0.29 & 0.45 \text { d } & 8.2 & 0.8 \\ 12-2,31-33 & 3.60 & 5.00 \mathrm{~d} & 0.22 & 0.30 \mathrm{~d} & 0.35 & 0.49 \mathrm{~d} & 10.3 & 0.6 \\ 13-2,30-33 & 4.20 & 6.00 & 0.31 & 0.44 & 0.32 & 0.46 & 13.1 & 1.0 \\ 13-3,109-112 & 4.46 & 6.49 & 0.36 & 0.52 & 0.29 & 0.42 & 15.4 & 1.2 \\ 13-4,144-145 & 3.57 & 5.02 & 0.28 & 0.39 & 0.31 & 0.44 & 11.5 & 0.6 \\ 14-1,66-70 & 5.60 & * & 0.31 & * & 0.38 & * & 14.7 & 0.8 \\ 14-2,6-8 & 5.36 & * & 0.15 & * & 0.40 & * & 13.4 & 0.4 \\ 15-1,53-55 & 6.72 & * & 0.19 & * & 0.38 & * & 17.7 & 0.5 \\ 15-1,90-91 & 6.03 & * & 0.92 & * & 0.43 & * & 14.0 & 2.1 \\ 15-1,100-101 & 5.53 & * & 1.36 & * & 0.50 & * & 11.1 & 2.7 \\ 15-1,148-150 & 5.00 & * & 1.19 & * & 0.38 & * & 13.2 & 3.13 \\ 16-2,8-10 & 8.59 & * & 0.23 & * & 1.13 & * & 7.6 & 0.20 \\ 16-2,122-123 & 7.34 & * & 0.01 & * & 1.61 & * & 4.6 & * \\ 16-2,123-124 & 7.30 & * & 0.53 & * & 1.35 & * & 5.8 & 0.4 \\ 16-3,77-80 & 6.10 & * & 1.00 & * & 0.43 & * & 14.2 & 2.3 \\ 20-2,40-50 & 5.79 & 9.90 & 0.15 & 0.26 & 0.63 & 1.08 & 9.2 & 0.2 \\ 23-1,10 & 10.75 & * & 0.12 & * & 2.32 & * & 4.6 & 0.05 \\ 23-3,23 & 10.92 & * & 0.18 & * & 2.74 & * & 4.0 & 0.1 \\ & & & & & & & & \\ 5110386 & & & & & & & & \end{array}$

Site 386

\begin{tabular}{|c|c|c|c|c|c|c|c|c|}
\hline $4-2,111-115$ & 6.15 & * & 0.11 & * & 1.06 & * & 5.8 & 0.1 \\
\hline $4-4,124-129$ & 4.74 & & 0.12 & & 1.11 & & 4.3 & 0.1 \\
\hline $5-2,46-50$ & 9.20 & & 0.19 & & 2.04 & & 4.5 & 0.1 \\
\hline $6-3,139-142$ & 0.99 & & 0.12 & & 0.10 & & 9.9 & 1.2 \\
\hline $6-4,128-133$ & 8.58 & & 0.21 & & 1.86 & & 4.6 & 0.1 \\
\hline $8-1,147-150$ & 7.01 & $8.25^{\mathrm{d}}$ & 0.15 & $0.18^{\mathrm{d}}$ & 1.70 & $2.00^{\mathrm{d}}$ & 4.1 & 0.1 \\
\hline $8-6,134-137$ & 9.45 & & 3.13 & & 2.30 & & 4.1 & 1.4 \\
\hline $9-3,127-130$ & 8.82 & & 0.21 & & 2.77 & & 3.2 & 0.1 \\
\hline $12-4,8-10$ & 7.26 & & 0.17 & & 1.37 & & 5.3 & 0.1 \\
\hline $13-3,127-133$ & 4.38 & $7.30^{\mathrm{d}}$ & 0.18 & $0.30^{\mathrm{d}}$ & 1.22 & $2.03^{\mathrm{d}}$ & 3.6 & 0.1 \\
\hline $14-1,1-5$ & 1.96 & & 0.04 & & 0.19 & & 10.3 & 0.2 \\
\hline $14-4,19-21$ & 5.43 & * & 0.04 & * & 0.35 & * & 15.5 & 0.1 \\
\hline $15-2,81-85$ & 2.08 & & 0.04 & & 0.19 & & 10.9 & 0.2 \\
\hline $17-1,132-135$ & 0.86 & & 0.04 & & 0.10 & & 8.6 & 0.4 \\
\hline $18-2,97-99$ & 1.35 & & 0.03 & & 0.05 & & 27.0 & 0.6 \\
\hline $28-2,123-125$ & 3.37 & & 0.07 & & 0.23 & & 14.6 & 0.3 \\
\hline $28-4,108-110$ & 2.23 & * & 0.02 & * & 0.20 & $*$ & $\begin{array}{l}14.0 \\
11.1\end{array}$ & 0.1 \\
\hline $30-2,89-91$ & & & 0.01 & $*$ & 0.14 & $*$ & 5.8 & 0.1 \\
\hline $30-6,59-61$ & $\begin{array}{l}.01 \\
1.39\end{array}$ & $1.76^{\mathrm{d}}$ & 0.11 & $0.14^{\mathrm{d}}$ & 0.13 & $0.16^{\mathrm{d}}$ & 10.7 & 0.8 \\
\hline $31-1,6-9$ & 3.07 & & 0.08 & & 0.22 & & $\begin{array}{l}10.7 \\
13.9\end{array}$ & 0.4 \\
\hline $34-2,25-27$ & 3.61 & & 0.04 & & 0.35 & & 10.3 & 0.1 \\
\hline $34-6,29-31$ & 4.74 & * & 0.25 & * & 0.36 & * & 13.2 & 0.7 \\
\hline $36-5,74-76$ & 5.49 & * & 0.51 & * & 0.45 & * & 12.2 & 1.1 \\
\hline $36-5,94-98$ & 5.92 & * & 0.47 & * & 0.44 & * & 13.4 & 1.1 \\
\hline $38-1,31-32$ & 3.13 & * & 0.47 & * & 0.22 & * & 14.2 & 2.1 \\
\hline $38-2,145-146$ & 4.40 & * & 0.54 & * & 0.25 & $*$ & 17.6 & 2.2 \\
\hline $38-2,149-150$ & 3.32 & * & 0.11 & * & 0.25 & & 13.3 & 0.4 \\
\hline $38-3,90-91$ & 4.16 & * & 0.21 & * & 0.30 & $*$ & 13.9 & 0.7 \\
\hline $38-4,127-128$ & 6.03 & * & 0.20 & * & 0.32 & * & 18.8 & 0.6 \\
\hline $39-1,129-131$ & 4.95 & * & 0.08 & * & 0.34 & * & 14.6 & 0.1 \\
\hline $39-1,142-143$ & 5.13 & * & 0.28 & * & 0.22 & * & 23.3 & 1.3 \\
\hline $39-1,144-145$ & 5.98 & $*$ & 0.76 & * & 0.29 & * & 20.6 & 2.7 \\
\hline $40-2,0-1$ & 2.75 & * & 0.15 & * & 0.21 & * & 13.1 & 0.7 \\
\hline $40-2,3-4$ & 6.48 & * & 0.36 & * & 0.24 & * & 27.0 & 1.5 \\
\hline $41-3,81-82$ & 4.53 & * & 0.04 & * & 0.27 & * & 16.8 & 0.1 \\
\hline $41-3,83-84$ & 3.91 & $*$ & 0.05 & * & 0.27 & $*$ & 14.5 & 0.1 \\
\hline $42-2,105-107$ & 0.85 & $*$ & 0.42 & * & 0.05 & * & 17.0 & 8.4 \\
\hline $44-3,23.5-24.5$ & 2.26 & * & 0.16 & * & 0.16 & * & 14.1 & 1.0 \\
\hline $45-5,84-88$ & 4.40 & & 0.14 & & 0.25 & & 17.6 & 0.6 \\
\hline
\end{tabular}

(see Table 1, Site 382, Cores 15 to 25; Site 385, Cores 14 to 23 ). In the great majority of samples, Fe content is greater than 5 per cent, values 6 to 9 per cent being common. The highest concentration (10.92 per cent)
TABLE 2 - Continued

\begin{tabular}{|c|c|c|c|c|c|c|c|c|}
\hline \multirow{2}{*}{$\begin{array}{c}\text { Sample } \\
\text { (Interval in } \mathrm{cm} \text { ) }\end{array}$} & \multicolumn{2}{|c|}{$\mathrm{Fe}$} & \multicolumn{2}{|c|}{$\mathrm{Mn}$} & \multicolumn{2}{|c|}{$\mathrm{Ti}$} & \multirow{2}{*}{$\begin{array}{l}\mathrm{Fe} / \mathrm{Ti} \\
\text { Ratio }\end{array}$} & \multirow{2}{*}{$\begin{array}{l}\mathrm{Mn} / \mathrm{Ti} \\
\text { Ratio }\end{array}$} \\
\hline & a & b & $\mathrm{a}$ & b & $\mathrm{a}$ & b & & \\
\hline $46-5,58-61$ & 4.44 & * & 0.02 & * & 0.26 & * & 17.1 & 0.1 \\
\hline $47-2,47-51$ & 5.14 & $*$ & 0.05 & * & 0.21 & * & 24.5 & 0.2 \\
\hline $47-4,47-50$ & 3.32 & * & 0.18 & * & 0.32 & * & 10.4 & 0.6 \\
\hline $48-3,123-124$ & 0.92 & & 0.75 & & 0.05 & & 18.4 & 15.0 \\
\hline $49-3,56-62$ & 2.80 & & 0.04 & & 0.20 & & 14.0 & 0.2 \\
\hline $49-3,90-93$ & 3.57 & 3.98 & 1.08 & 1.20 & 0.19 & 0.21 & 18.8 & 5.7 \\
\hline $50-3,35-36$ & 2.14 & 7.13 & 1.94 & 6.47 & 0.10 & 0.33 & 21.4 & 19.4 \\
\hline $51-2,68-69$ & 3.79 & & 0.18 & & 0.20 & & 10.0 & 0.9 \\
\hline $53-1,120-121$ & 2.63 & 3.36 & 1.22 & 1.56 & 0.16 & 0.20 & 16.4 & 7.6 \\
\hline $53-3,87-89$ & 1.85 & 6.58 & 1.02 & 3.63 & 0.10 & 0.36 & 18.5 & 10.2 \\
\hline $54-4,99-100$ & 3.97 & 7.94 & 0.40 & 0.80 & 0.14 & 0.28 & 28.4 & 2.8 \\
\hline $54-4,100-101$ & 3.10 & 7.18 & 0.83 & 1.92 & 0.21 & 0.49 & 14.8 & 3.9 \\
\hline $55-2,60-61$ & 1.96 & 4.46 & 0.86 & 1.96 & 0.11 & 0.25 & 17.8 & 7.8 \\
\hline $55-5,143-144$ & 2.54 & 5.01 & 0.56 & 1.10 & 0.10 & 0.20 & 25.4 & 5.6 \\
\hline $57-6,23-26$ & 2.33 & 4.99 & 0.86 & 1.84 & 0.11 & 0.24 & 23.3 & 7.8 \\
\hline $58-6,51-52$ & 5.38 & 6.60 & 2.68 & 3.29 & 0.17 & 0.21 & 31.6 & 15.8 \\
\hline $59-4,57-60$ & 3.33 & 5.18 & 3.69 & 5.74 & 0.13 & 0.20 & 25.6 & 28.3 \\
\hline $60-5,140-141$ & 4.24 & $*$ & 0.18 & $*$ & 0.22 & $*$ & 19.3 & 0.8 \\
\hline $\begin{array}{l}03-4,19-23 \\
63-41\end{array}$ & $\begin{array}{r}4.24 \\
22.94\end{array}$ & * & $\begin{array}{l}0.18 \\
5.99\end{array}$ & * & 0.13 & $*$ & $\begin{array}{l}17.3 \\
176.5\end{array}$ & $\begin{array}{r}0.0 \\
46.0\end{array}$ \\
\hline $\begin{array}{l}63-4,29-23 \\
64-2,15-16\end{array}$ & $\begin{array}{r}22.94 \\
7.94\end{array}$ & * & $\begin{array}{l}.999 \\
0.02\end{array}$ & * & 0.17 & * & 46.7 & $\begin{array}{r}46.0 \\
0.1\end{array}$ \\
\hline $64-2,17-18$ & 7.14 & * & 0.02 & * & 0.23 & * & 31.0 & 0.1 \\
\hline $64-2,47-48$ & 9.36 & & 0.05 & & 0.17 & & 55.1 & 0.3 \\
\hline $64-2,79-80$ & 20.46 & * & 11.41 & * & 0.08 & * & 255.7 & 142.5 \\
\hline $64-3,22-23$ & $\begin{array}{r}4.40 \\
4.92\end{array}$ & & 0.04 & & 0.22 & & 22.4 & 0.2 \\
\hline $64-3,24-25$ & 8.28 & * & 0.02 & * & 0.02 & * & 41.4 & 0.1 \\
\hline $64-3,50-51$ & 8.41 & & 0.07 & & 0.17 & & 49.4 & 0.4 \\
\hline $64-4,75-76$ & 8.58 & * & 0.03 & * & 0.22 & * & 39.0 & 0.1 \\
\hline $65-3,104-105$ & 9.46 & * & 0.05 & * & 0.25 & * & 37.8 & 0.2 \\
\hline \multicolumn{9}{|l|}{ Site 387} \\
\hline $1-6,80-82$ & 5.44 & * & 0.08 & * & 0.51 & * & 10.7 & 0.2 \\
\hline $2-2,80-82$ & 4.38 & * & 0.30 & * & 0.44 & * & 10.0 & 0.7 \\
\hline $3-2,130-132$ & $\begin{array}{l}3.96 \\
\end{array}$ & * & 0.31 & * & 0.49 & * & 8.0 & 0.6 \\
\hline $6-3,70-73$ & 3.84 & * & 0.24 & * & 0.34 & $*$ & 11.3 & 0.7 \\
\hline $7-3,80-83$ & 2.41 & $*$ & 0.45 & * & 0.25 & * & 9.6 & 1.8 \\
\hline $10-1,146-150$ & 1.77 & & 0.05 & & 0.19 & & 9.3 & 0.3 \\
\hline $13-1,104-106$ & 3.55 & 3.94 & 0.04 & 0.04 & 0.34 & 0.38 & 10.4 & 0.1 \\
\hline $16-3,56-60$ & $\begin{array}{l}3.53 \\
1.57\end{array}$ & $\begin{array}{l}1.94 \\
1.96 \mathrm{~d}\end{array}$ & $\begin{array}{l}0.04 \\
0.19\end{array}$ & $\begin{array}{l}0.04 \\
0.24\end{array}$ & $\begin{array}{l}0.34 \\
0.12\end{array}$ & $\begin{array}{l}0.38 \\
0.15\end{array}$ & $\begin{array}{l}10.4 \\
13.1\end{array}$ & $\begin{array}{l}.1 \\
1.6\end{array}$ \\
\hline $23-4,120-122$ & 4.15 & & 0.21 & & 0.32 & & 13.0 & 0.7 \\
\hline $25-3,144-146$ & 1.50 & * & & * & 0.15 & * & 10.0 & 0.1 \\
\hline $\begin{array}{l}26-3,144-146 \\
26-2,0-2\end{array}$ & $\begin{array}{l}1.5 \\
4.2\end{array}$ & * & $\begin{array}{l}0.02 \\
0.02\end{array}$ & * & 0.31 & * & $\begin{array}{l}10.0 \\
13.5\end{array}$ & $\begin{array}{l}0.1 \\
0.1\end{array}$ \\
\hline $27-1,39-41$ & 2.77 & * & 0.01 & * & 0.30 & * & 9.2 & 0.1 \\
\hline $27-6,40-42$ & 1.66 & 3.38 & 0.09 & 0.18 & 0.24 & 0.49 & 6.9 & 0.4 \\
\hline $28-1,131-133$ & $\begin{array}{l}2.00 \\
2.67\end{array}$ & 4.40 & 0.22 & 0.36 & 0.21 & 0.39 & 12.7 & 1.0 \\
\hline $29-2,12-15$ & 5.78 & $*$ & 0.08 & $*$ & 0.48 & $*$ & 12.0 & 0.2 \\
\hline $29-4,101-106$ & 5.54 & $*$ & $\begin{array}{l}0.53 \\
0.03\end{array}$ & * & 0.40 & * & 19. & 1.3 \\
\hline $30-1,134-140$ & 3.02 & $*$ & 0.01 & * & 0.12 & * & 25.2 & 0.1 \\
\hline $2,40-43$ & 2.9 & * & 0.01 & * & 0.2 & * & 13 & 0.04 \\
\hline $2,103-106$ & 4.6. & * & 0.02 & * & 0.19 & * & 24 & 0.1 \\
\hline $35-2,27-29$ & 1.27 & * & 0.02 & * & 0.10 & * & 12.7 & 0.2 \\
\hline $35-5,0-2$ & 2.76 & * & 0.01 & * & 0.32 & * & 8 & 0.03 \\
\hline $36-1,71-73$ & 3.62 & * & 0.01 & * & 0.31 & * & 11.8 & 0.02 \\
\hline $37-2,101-103$ & 3.04 & * & 0.02 & * & 0.22 & * & 13.8 & 0.1 \\
\hline $37-3,101-103$ & 1.69 & & 1.01 & & 0.06 & & 28.2 & 16.8 \\
\hline $39-2,112-114$ & 1.11 & 4.46 & 0.03 & 0.12 & 0.07 & 0.28 & 15.8 & 0.4 \\
\hline $40-2,52-54$ & 2.68 & 8.14 & 0.02 & 0.06 & 0.10 & 0.30 & 26.8 & 0.1 \\
\hline $42-1,72-75$ & 2.41 & 6.39 & 0.02 & 0.05 & 0.13 & 0.34 & 18.5 & 0.1 \\
\hline $44-1,71-75$ & 0.36 & 3.77 & 0.05 & 0.52 & $\operatorname{tr}$ & - & - & - \\
\hline $45-1,142-145$ & 0.66 & & 0.08 & & tr & - & - & - \\
\hline $46-1,86-88$ & 0.34 & 6.18 & 0.03 & 0.54 & tr & - & - & - \\
\hline $47-1,143-145$ & 0.47 & 13.80 & 0.04 & 1.17 & tr & - & - & - \\
\hline $48-1,119-120$ & $\begin{array}{l}0.47 \\
0.49\end{array}$ & $\begin{array}{r}11.80 \\
8.30\end{array}$ & $\begin{array}{l}0.04 \\
0.02\end{array}$ & 0.34 & $\begin{array}{l}\text { tr } \\
\text { tr }\end{array}$ & - & - & - \\
\hline & 3.01 & 7.39 & 0.04 & 0.10 & 0.13 & 0.39 & 23.2 & 0.3 \\
\hline $50-1,35-38$ & 5.24 & & 0.04 & & 0.62 & & 8.4 & 0.1 \\
\hline
\end{tabular}

${ }^{\mathrm{a}}$ Bulk sediment.

${ }^{b}$ Calculated on a $\mathrm{CaCO}_{3}$-free basis.

$\mathrm{c}_{*}=\mathrm{CaCO}_{3}$ less than $10 \%$.

${ }^{\mathrm{d}} \mathrm{CaCO}_{3}$ content in the sample was not determined, the closest "carbonate bomb" or "carboncarbonate" determination was used for $\mathrm{CaCO}_{3}$-free basis calculation.

$\mathrm{e}_{\mathrm{tr}}=$ trace.

was determined in an altered volcaniclastic breccia in Section 385-23-3. The most variable iron contents were found in the variegated intervals (Subunits 3A, 3C, and $3 \mathrm{E}$ at Site 382 ) where they range from 3.9 per cent in light layers to 10 per cent in dark reddish brown layers. Some low values in these intervals increase when analyses are calculated on $\mathrm{CaCO}_{3}$-free basis.

Volcaniclastic turbidites of Site 386 (Subunit 3B) are also enriched in iron; concentrations up to 9.5 per cent were determined in the heavy mineral sandstones and siltstones.

Iron content in the red claystone intervals at Sites 386 (Unit 5) and 387 (Unit 5) is only slightly higher than in "normal" hemipelagic clay. The highest val- 
ues, determined in reddish brown layers, are 5 to 6 per cent, whereas gray layers are lower in iron.

Greatly variable iron content was found in the Lower Cretaceous black and greenish gray claystones at Sites 386 and 387. It ranges from 0.85 per cent in highly siliceous samples to 22.9 per cent in sideritic interbeds. It is noteworthy that the claystones at Site 387 , as well as in the upper part of Unit 6 (Cores 42 to 49 ) of Site 386 are relatively low in iron. High concentrations occur mostly in the lower part of Unit 6 of Site 386 , beginning with Core 50, but especially in Cores 63 to 65 , directly above basaltic basement. In Cores 63 to 65 (Table 2 ) only rare analyses show less than 5 per cent of $\mathrm{Fe}$, values 7 to 9 per cent being most common. Siderite is probably the most important iron-bearing mineral in this interval, as shown by phase-analysis data (Table 3), where iron carbonate appears as "soluble" bivalent Fe. Pyrite also carries an essential portion of iron in some samples.

Calcareous sediments, that is, nannofossil oozes of Site 384, limestones of Site 387, marly chalk of Unit 4, Site 387 , and other calcareous interlayers, are low in iron owing to dilution by biogenic $\mathrm{CaCO}_{3}$. In general, $\mathrm{Fe}$ content decreases concomitantly with increasing $\mathrm{CaCO}_{3}$. If calculated on a carbonate-free basis, the iron content is still low throughout Site 384 ( 2 to 4.37 per cent, Table 1), but relatively high values occur in the marly ooze of Subunit 2C, Site 385, and in the limestones of Unit 7. Non-calcareous residue of the latter contains up to 13.8 per cent $\mathrm{Fe}$, which occurs most likely as pyrite, and partly as siderite. The accuracy of determination is, however, not sufficient to make certain conclusions about systematic enrichment in $\mathrm{Fe}$ toward basaltic basement.

\section{Manganese}

Manganese was determined in the same samples as iron (Table 2). In general, manganese concentrations are low, less than 0.2 per cent, the limit usually applied to separate "non-manganese" from "low-manganese" sediments. Mn content is less than 0.1 per cent (with rare exceptions) in Pleistocene-Pliocene turbidites (Site 382), in Miocene hemipelagic clay (Sites 382 and 385), and in Eocene to Paleocene siliceous and calcareous turbidites. All these sediments display features of reducing conditions of diagenesis and soluble $\mathrm{Mn}$ has probably been leached out.

In the red-and brown-colored claystones, as well as in volcaniclastic sediment, low Mn values predominate, although several layers are enriched in manganese: up to 0.76 per cent in the variegated claystone of Site 382 ; up to 1.36 per cent in vitric silty clay of Site 385 (Core 15 , Section 1; see Table 2); up to 0.76 per cent in a dark interbed in the red claystone unit of Site 386 (Core 39), and up to 0.53 per cent in red claystone of Site 387 (Core 29).

Unusually variable manganese content was found in black and greenish gray claystones, where very low values $(0.2$ to $0.03 \%)$, normal in reduced sediments, alternate with concentrations of up to 11.4 per cent. The
TABLE 3

Fe Phase Analysis Data for Leg 43 Sediments

\begin{tabular}{|c|c|c|c|c|c|c|c|c|c|c|}
\hline \multirow{3}{*}{$\begin{array}{c}\text { Sample } \\
\text { (Interval in } \mathrm{cm} \text { ) }\end{array}$} & \multirow{3}{*}{$\begin{array}{c}\mathrm{Fe} \\
\text { Total }\end{array}$} & \multicolumn{4}{|c|}{$\mathrm{Fe}^{+3}$} & \multicolumn{4}{|c|}{$\mathrm{Fe}^{+2}$} & \multirow{3}{*}{$\begin{array}{c}\mathrm{Fe} \\
\text { in } \\
\text { Pyrite }\end{array}$} \\
\hline & & \multicolumn{2}{|c|}{ Insoluble } & \multicolumn{2}{|c|}{ Soluble } & \multicolumn{2}{|c|}{ Insoluble } & \multicolumn{2}{|c|}{ Soluble } & \\
\hline & & a & b & $\mathrm{a}$ & b & a & b & $\mathrm{a}$ & b & \\
\hline \multicolumn{11}{|l|}{ Site 382} \\
\hline $1-4,113-115$ & 1.39 & 0.31 & 22.1 & 0 & 0 & 1.09 & 77.9 & 0 & 0 & 0 \\
\hline $7-2,81-85$ & 5.00 & 3.92 & 78.2 & 0 & 0 & 1.09 & 21.8 & 0 & 0 & 0 \\
\hline $15-5,10-12$ & 5.00 & 4.18 & 83.4 & 0.42 & 8.4 & 0.41 & 8.2 & 0 & 0 & 0 \\
\hline $15-6,44-47^{c}$ & 4.43 & 3.74 & 84.2 & 0.43 & 9.7 & 0.27 & 6.1 & 0 & 0 & 0 \\
\hline $15-6,44-47^{d}$ & 4.16 & 4.16 & 10.0 & 0 & 0 & 0 & 0 & 0 & 0 & 0 \\
\hline $15-6,60-62$ & 3.88 & 3.46 & 89.2 & 10.8 & 0 & 0 & 0 & 0 & 0 & 0 \\
\hline $16-1,0-3$ & 6.10 & 3.84 & 62.8 & 2.14 & 35.1 & 0.12 & 2.0 & 0 & 0 & 0 \\
\hline $16-1,94-98$ & 5.00 & 4.22 & 84.4 & 0.78 & 15.6 & 0 & 0 & 0 & 0 & 0 \\
\hline $16-6,45-48$ & 10.00 & 4.20 & 42.0 & 5.80 & 58.0 & 0 & 0 & 0 & 0 & 0 \\
\hline $16-6,123-124$ & 8.59 & 5.24 & 60.9 & 3.36 & 39.1 & 0 & 0 & 0 & 0 & 0 \\
\hline $18-1,61-65$ & 6.72 & 1.69 & 25.1 & 4.91 & 73.1 & 0.12 & 1.8 & 0 & 0 & 0 \\
\hline $18-2,4-9$ & 3.92 & 1.89 & 48.2 & 1.47 & 37.5 & 0.56 & 1.4 & 0 & 0 & 0 \\
\hline $18-2,109-112$ & 5.88 & 3.40 & 57.8 & 2.36 & 40.1 & 0.12 & 2.0 & 0 & 0 & 0 \\
\hline $18-4,9-12$ & 6.44 & 3.76 & 58.4 & 2.41 & 37.4 & 0.27 & 4.2 & 0 & 0 & 0 \\
\hline $19-4,20-25$ & 7.49 & 4.66 & 62.0 & 1.85 & 24.6 & 1.00 & 13.3 & 0 & 0 & 0 \\
\hline $20-2,36-38$ & 8.32 & 4.83 & 58.0 & 2.80 & 33.6 & 0.69 & 8.3 & 0 & 0 & 0 \\
\hline $21-3,82-84$ & 7.68 & 3.64 & 47.4 & 1.68 & 21.9 & 1.86 & 24.2 & 0 & 0 & 0 \\
\hline $22-2,86-89$ & 6.63 & 3.86 & 58.2 & 0 & 0 & 0.97 & 15.4 & 1.80 & 27.1 & 0 \\
\hline $24-1,90-93$ & 6.37 & 3.10 & 48.7 & 0.49 & 7.7 & 0.54 & 8.5 & 0 & 0 & 0 \\
\hline $25, \mathrm{CC}$ & 5.00 & 2.57 & 49.1 & 1.26 & 24.0 & 1.40 & 26.8 & 0 & 0 & 0 \\
\hline \multicolumn{11}{|l|}{ Site 384} \\
\hline $4-6,114-115$ & 1.66 & 1.66 & 100 & 0 & 0 & 0 & 0 & 0 & 0 & 0 \\
\hline \multicolumn{11}{|l|}{ Site 385} \\
\hline $3-2,130-132$ & 4.43 & 3.89 & 87.8 & 0 & 0 & 0.54 & 12.2 & 0 & 0 & 0 \\
\hline $8-5,130-132$ & 3.60 & 3.48 & 96.7 & 0 & 0 & 0.12 & 3.3 & 0 & 0 & 0 \\
\hline $12-2,31-33$ & 3.60 & 3.27 & 90.8 & 0 & 0 & 0.33 & 9.2 & 0 & 0 & 0 \\
\hline $14-1,66-70$ & 5.60 & 4.71 & 84.0 & 0.63 & 11.2 & 0.27 & 4.8 & 0 & 0 & 0 \\
\hline $15-1,53-55$ & 6.72 & 5.22 & 77.7 & 1.37 & 20.4 & 0.12 & 1.8 & 0 & 0 & 0 \\
\hline $15-1,100-101$ & 5.53 & 5.53 & 100 & 0 & 0 & 0 & 0 & 0 & 0 & 0 \\
\hline $15-1,148-150$ & 5.00 & 4.30 & 86.0 & 0.63 & 12.6 & 0 & 0 & 0 & 0 & 0 \\
\hline $16-2,8-10$ & 8.59 & 6.94 & 80.7 & 1.54 & 17.9 & 0.12 & 1.4 & 0 & 0 & 0 \\
\hline $16-3,77-80$ & 6.10 & 4.70 & 77.0 & 1.40 & 23.0 & 0 & 0 & 0 & 0 & 0 \\
\hline $20-2,40-50$ & 5.79 & 3.02 & 52.2 & 2.66 & 45.9 & 0.12 & 2.1 & 0 & 0 & 0 \\
\hline \multicolumn{11}{|l|}{ Site 386} \\
\hline $34-2,25-27$ & 3.61 & 3.33 & 92.2 & 0 & 0 & 0.28 & 7.8 & 0 & 0 & 0 \\
\hline $36-5,74-76$ & 5.49 & 5.06 & 92.2 & 0.43 & 7.8 & 0 & 0 & 0 & 0 & 0 \\
\hline $38-2,145-146$ & 4.40 & 4.40 & 100 & 0 & 0 & 0 & 0 & 0 & 0 & 0 \\
\hline $38-2,149-150$ & 3.32 & 3.21 & 96.7 & 0 & 0 & 0.11 & 3.3 & 0 & 0 & 0 \\
\hline $39-1,129-131$ & 4.71 & 2.70 & 57.3 & 2.00 & 42.5 & 0 & 0 & 0 & 0 & 0 \\
\hline $45-5,84-88$ & 4.40 & 3.90 & 88.6 & 0 & 0 & 0.50 & 11.4 & 0 & 0 & 0 \\
\hline $47-4,47-50$ & 3.32 & 2.33 & 70.2 & 0 & 0 & 0 & 0 & 0.87 & 26.2 & 0.12 \\
\hline $49-3,56-62$ & 2.80 & 2.03 & 72.5 & 0 & 0 & 0.77 & 27.5 & 0 & 0 & 0 \\
\hline $63-4,19-23$ & 22.94 & 0 & 0 & 0 & 0 & 0 & 0 & 22.47 & 98.0 & 0.47 \\
\hline $64-2,47-48$ & 9.36 & 7.61 & 81.3 & 0 & 0 & 0.85 & 9.1 & 0.80 & 8.5 & 0.10 \\
\hline $64-2,79-80$ & 20.46 & 0 & 0 & 0 & 0 & 0 & 0 & 20.06 & 98.0 & 0.40 \\
\hline $65-3,104-105$ & 9.46 & 0 & 0 & 0 & 0 & 0 & 0 & 9.40 & 99.4 & 0.07 \\
\hline \multicolumn{11}{|l|}{ Site 387} \\
\hline $23-4,120-122$ & 4.15 & 3.93 & 94.7 & 0 & 0 & 0.22 & 5.3 & 0 & 0 & 0 \\
\hline $27-1,39-41$ & 2.77 & 2.50 & 90.2 & 0 & 0 & 0.27 & 9.8 & 0 & 0 & 0.45 \\
\hline $27-6,40-42$ & 1.66 & 1.27 & 76.5 & 0 & 0 & 0.27 & 16.3 & 0 & 0 & 0.22 \\
\hline $28-1,131-133$ & 2.67 & 2.40 & 89.9 & 0 & 0 & 0.27 & 10.1 & 0 & 0 & 0 \\
\hline $29-2,12-15$ & 5.78 & 3.30 & 57.1 & 1.18 & 20.4 & 0.12 & 2.1 & 0 & 0 & 0 \\
\hline $29-4,101-106$ & 5.54 & 4.04 & 72.9 & 1.26 & 22.7 & 0.27 & 4.9 & 0 & 0 & 0 \\
\hline $30-1,134-140$ & 3.02 & 3.02 & 100 & 0 & 0 & 0 & 0 & 0 & 0 & 0 \\
\hline $35-5,0-2$ & 2.76 & 2.21 & 80.1 & 0 & 0 & 0.55 & 19.9 & 0 & 0 & 0 \\
\hline
\end{tabular}

latter are related to the authigenic manganese carbonates (rhodochrosite, manganic siderite, and manganic calcite) identified by X-ray and optical methods. A high-manganese interval occurs in Unit 6 of Site 386, comprising Cores 49 to 59 (especially 53 to 59 ); it is separated from basaltic basement by an interval of high iron, but lower Mn concentrations.

\section{Titanium}

Titanium was determined in the same samples as $\mathrm{Fe}$ and $\mathrm{Mn}$. The results can therefore be used to calculate $\mathrm{Fe} / \mathrm{Ti}$ and $\mathrm{Mn} / \mathrm{Ti}$ ratios in order to evaluate relative enrichment of these elements with respect to titanium, which is supposed to be geochemically less mobile during both sedimentation and diagenesis. 
Titanium content is uniform in the Miocene hemipelagic clay (Sites 382 and 385 ) ranging from 0.4 to 0.58 per cent (Table 2). Only a few lower values appear in the Pleistocene-Pliocene terrigenous turbidites at Site 382 , where these can be interpreted as dilution by biogenic $\mathrm{CaCO}_{3}$ and by quartzose sand or silt. Low Ti ( 0.14 to 0.37 per cent) was determined in radiolarian mud and zeolitic silty clay of Unit 2, Site 385 . Eocene siliceous turbidites of Sites 386 and 387 are low in titanium ( 0.12 to 0.35 per cent) where the "normal" $\mathrm{Ti}$ concentration in terrigenous clay is diluted by biogenic and authigenic silica.

Volcanogenic sediments of Sites 382 and 385, as well as volcaniclastic turbidites of Site 386 , Subunit 3B, are marked by high titanium content, which usually ranges from 1 to 3 per cent (Table 2). The volcaniclastic breccia shows the same range of concentration. It should be noted, however, that high Ti does not occur in the uppermost portions of variegated claystone at Site 382 (Cores 15 to $16-1,0-3 \mathrm{~cm}$ ), as well as in the upper "vitric silty clay" at Site 385 (Cores 14 through 15 ). These fine-grained sediments are either not volcanogenic, or were formed of volcaniclastic matter low in titanium.

The red claystone intervals at Sites 386 and 387 display "normal" or low Ti content. Black and greenish gray claystones at the same sites are low in titanium; very low values ( 0.05 to 0.2 per cent) are common, and probably reflect dilution of Ti-bearing terrigenous matter by authigenic silicification, or partly by biogenic silica and organic matter.

Extremely low $\mathrm{Ti}$ was detected in calcareous sediments, both in nannofossil oozes at Site 384 (0.04 to 0.18 per cent) and in Lower Cretaceous limestones at Site 387 (trace to 0.62 per cent). If calculated on $\mathrm{CaCO}_{3}$-basis, "normal"' or lower values appear.

$\mathrm{The} \mathrm{Fe} / \mathrm{Ti}$ ratio ranges from 2.5 to 46.7 . Two yet higher values (176 and 255) were found in sideritic interbeds. Hemipelagic clay (Sites 382 and 385) is characterized by a ratio of 8.9 to 14.1. Some lower values occur in terrigenous turbidites of Site 382; these belong to the low-iron, quartzose interbeds. The $\mathrm{Fe} / \mathrm{Ti}$ ratio in Eocene to Paleocene siliceous and calcareous turbidites, marly oozes, and radiolarian muds is similar to that in hemipelagic clay, indicating a virtually constant composition for the terrigenous matter, and the absence of any additional iron or titanium. With a few exceptions, the same is true for Site 384 nannofossil ooze.

A sharp decrease of the ratio marks the transition from "normal" sediments to the sediments with a volcaniclastic component at Sites 382, 385, and 386. Values less than 9 predominate, except in the upper portion of the variegated claystone sequence at Site 382 (Cores 15 to $16-1,0-3 \mathrm{~cm}$ ), where the $\mathrm{Fe} / \mathrm{Ti}$ ratio ranges from 12.2 to 7.7 , and in the upper "vitric silty clay" at Site 385 (Cores 14, 15), where the ratio is 11.1 to 17.7. These exceptions point once more to a difference between the upper intervals and other volcaniclastic sequences. The lowest values (less than 4) were found in some "rusty" or gray, strongly altered volcaniclastic layers on the high level of both $\mathrm{Fe}$ and Ti concentration, whereas volcaniclastic breccia is characterized by a ratio of 5 to 8 .

In the red claystone intervals at Sites 386 (Cores 36 to 41 ) and 387 (Core 29) $\mathrm{Fe} / \mathrm{Ti}$ ratio is rather high. The values, however, are considerably lower than those found in oceanic "metalliferous" sediments.

Variable, but in many cases very high values of the $\mathrm{Fe} / \mathrm{Ti}$ ratio occur in the black and greenish gray claystone units at Sites 386 and 387. Obviously, some additional iron (as compared with that in the primary terrigenous material) is included in the sediments. It seems important, that the ratio tends to increase downward toward underlying basalt at Site 387 . Below Core 58 all values are higher than 25 . In the modern ocean the ratio is this high only in "metalliferous" sediments.

$\mathrm{The} \mathrm{Fe} / \mathrm{Ti}$ ratio is relatively high in the Lower Cretaceous limestones at Site 387, which overlie basaltic basement. However, it was determined here on a very low level of both $\mathrm{Fe}$ and $\mathrm{Ti}$ concentration.

The Mn/Ti ratio is low (0.1-0.2 or less) throughout the section at Site 382. Rare, high values (up to 3.6) are related to local Mn concentrations in Cores 6 and 7 (Miocene hemipelagic clay) and in Cores 15 and 16 (variegated claystone). At Site 385, below the hemipelagic clay, a pelagic radiolarian mud and marly ooze interval has a higher $\mathrm{Mn} / \mathrm{Ti}$ ratio, which ranges from 0.3 to 1.6 . Relatively high values were detected in the upper "vitric silty clay" (0.4-3.1, Cores 14 and 15 , Table 2).

In the pelagic calcareous sequence at Site 384 , the $\mathrm{Mn} / \mathrm{Ti}$ ratio ranges from 0.3 to 3.4 , showing relative enrichment in $\mathrm{Mn}$ versus $\mathrm{Ti}$, as is usual in oxidized pelagic sediments. The same patterns appear in the red claystones of Sites 386 and 387, where the ratio ranges from 0.1 to 2.7 .

Extreme variations in the $\mathrm{Mn} / \mathrm{Ti}$ ratio (from 0.1 to 142.5 ) occur in the black and greenish gray claystone units at Sites 386 and 387 . Values are generally low; a few very high values are related to authigenic manganese carbonates. A distinct interval of very high $\mathrm{Mn} / \mathrm{Ti}$ ratio appear at Site 386, Cores 49 to 63 , where it ranges from 0.8 to 46 .

\section{Aluminum \\ Aluminum was determined in selected samples (Ta- ble 4) mainly in order to calculate $\mathrm{Fe} / \mathrm{Al}$ and $\mathrm{Mn} / \mathrm{Al}$ ratios, which are supposed to be most indicative in dis- tinguishing "metalliferous" sediments. $\mathrm{Al} / \mathrm{Ti}$ ratio is usually significant as a measure of basic volcaniclastic admixture in normal sediments. \\ The $\mathrm{Fe} / \mathrm{Al}$ ratio in the whole set of analyzed sam- ples ranges from 0.3 to 12.8 . Two extremely high val- ues belong to the sideritic interbeds of Site 386, Unit 6; other values fall in the limits 0.3 to 2.9 . Volcaniclastic sediments with higher values of 0.8 to 1.8 , stand out against background values of 0.4 to 0.6 . In the volcani- clastic breccia of Site 385 values up to 2.9 are reached. Again, these features are absent in the upper portions of volcanogenic units of Sites 382 (Cores 15 to 16, Sec-}


TABLE 4

Aluminum Content and Characteristic Ratios With Fe, Mn, Ti for Leg 43 Sediments

\begin{tabular}{lllll}
\hline $\begin{array}{c}\text { Sample } \\
\text { (Interval in } \mathrm{cm})\end{array}$ & $\mathrm{Al}$ & $\mathrm{Fe} / \mathrm{Al}$ & $\mathrm{Mn} / \mathrm{Al}$ & $\mathrm{Al} / \mathrm{Ti}$ \\
\hline
\end{tabular}

Site 382

$\begin{array}{lrlll}1-4,113-115 & 5.1 & 0.3 & 0.01 & 20.4 \\ 1-5,110-112 & 7.0 & 0.6 & 0.01 & 15.6 \\ 3-1,126-128 & 8.2 & 0.6 & 0.01 & 17.8 \\ 7-2,81-83 & 10.2 & 0.5 & 0.01 & 20.4 \\ 11-1,96-98 & 9.4 & 0.6 & 0.01 & 20.4 \\ 15-4,147-150 & 9.8 & 0.6 & 0.01 & 20.8 \\ 15-6,44-47 & 9.3 & 0.5 & 0.02 & 37.2 \\ 15-6,44-47 & 9.5 & 0.4 & 0.06 & 31.0 \\ 15-6,60-62 & 9.3 & 0.4 & 0.08 & 37.2 \\ 16-1,0-3 & 10.4 & 0.6 & 0.01 & 20.8 \\ 16-6,45-48 & 8.3 & 1.2 & 0.03 & 5.8 \\ 16-6,123-126 & 8.2 & 1.0 & 0.03 & 5.5 \\ 18-1,61-65 & 8.6 & 0.8 & 0.03 & 7.5 \\ 18-2,4-9 & 5.3 & 0.7 & 0.02 & 7.0 \\ 18-2,109-112 & 6.5 & 0.9 & 0.02 & 6.0 \\ 18-4,9-12 & 6.5 & 1.0 & 0.02 & 6.4 \\ 20-2,36-38 & 9.2 & 0.9 & 0.02 & 8.1 \\ 20-5,55-57 & 6.5 & 0.3 & 0.03 & 7.3 \\ 21-3,82-84 & 8.5 & 0.9 & 0.03 & 6.8 \\ 22-2,86-89 & 8.2 & 0.8 & 0.02 & 4.8 \\ 24-1,90-93 & 6.4 & 1.0 & 0.03 & 5.9 \\ 25-2,49-51 & 5.9 & 1.5 & 0.02 & 2.4 \\ 25, \text { CC } & 4.4 & 1.1 & 0.02 & 7.0\end{array}$

Site 385

$\begin{array}{lrrrr}3-2,130-132 & 9.4 & 0.5 & 0.01 & 23.5 \\ 8-5,130-132 & 8.6 & 0.4 & 0.02 & 34.4 \\ 10-1,138-140 & 5.4 & 0.4 & 0.02 & 27.0 \\ 11-2,24-26 & 7.8 & 0.6 & 0.07 & 21.1 \\ 12-2,31-33 & 6.4 & 0.6 & 0.03 & 18.3 \\ 13-3,109-112 & 7.0 & 0.6 & 0.05 & 24.1 \\ 14-1,66-70 & 10.5 & 0.5 & 0.03 & 27.6 \\ 14-2,6-8 & 10.0 & 0.5 & 0.02 & 25.0 \\ 15-1,53-55 & 10.9 & 0.6 & 0.02 & 28.7 \\ 15-1,100-101 & 12.9 & 0.4 & 0.10 & 25.8 \\ 15-1,148-150 & 10.9 & 0.5 & 0.11 & 28.7 \\ 16-2,8-10 & 10.9 & 0.8 & 0.02 & 9.6 \\ 16-2,122-123 & 8.4 & 0.8 & 0.01 & 5.2 \\ 16-3,77-80 & 4.5 & 1.4 & 0.22 & 10.5 \\ 20-2,40-50 & 10.8 & 0.5 & 0.01 & 17.1 \\ 23-1,10 & 6.1 & 1.8 & 0.02 & 2.6 \\ 23-3,23 & 3.7 & 2.9 & 0.05 & 1.4\end{array}$

Site 386

\begin{tabular}{lrrrr}
$4-2,111-115$ & 7.9 & 0.8 & 0.01 & 7.4 \\
$6-4,128-133$ & 5.5 & 1.6 & 0.04 & 3.0 \\
$8-1,147-150$ & 6.0 & 1.2 & 0.02 & 3.5 \\
$8-6,134-137$ & 5.1 & 1.8 & 0.61 & 2.2 \\
$9-3,127-130$ & 5.5 & 1.6 & 0.04 & 2.0 \\
$13-3,127-133$ & 3.0 & 1.5 & 0.06 & 2.5 \\
$14-4,19-21$ & 6.6 & 0.8 & 0.01 & 18.8 \\
$28-2,123-125$ & 5.2 & 0.6 & 0.01 & 22.6 \\
$28-4,108-110$ & 4.3 & 0.5 & 0.01 & 21.5 \\
$30-2,89-91$ & 3.0 & 0.3 & 0.01 & 21.4 \\
$30-6,59-61$ & 4.2 & 0.3 & 0.03 & 32.3 \\
$31-1,6-9$ & 5.8 & 0.5 & 0.01 & 26.4 \\
$34-2,25-27$ & 7.5 & 0.5 & 0.01 & 21.4 \\
$34-6,29-31$ & 7.6 & 0.6 & 0.03 & 21.1 \\
$36-5,74-76$ & 10.7 & 0.5 & 0.05 & 23.8 \\
$36-5,94-98$ & 10.4 & 0.6 & 0.04 & 23.6 \\
$38-1,31-32$ & 4.8 & 0.6 & 0.10 & 21.8 \\
$38-2,145-146$ & 6.3 & 0.7 & 0.09 & 25.2 \\
$38-2,149-150$ & 6.0 & 0.5 & 0.02 & 24.0 \\
\hline
\end{tabular}

TABLE 4 - Continued

\begin{tabular}{lrrrr}
\hline $\begin{array}{l}\text { Sample } \\
\text { (Interval in cm) }\end{array}$ & Al & Fe/Al & Mn/A1 & Al/Ti \\
\hline $38-3,90-91$ & 5.6 & 0.7 & 0.04 & 18.7 \\
$39-1,129-131$ & 7.1 & 0.7 & 0.01 & 20.9 \\
$39-1,144-145$ & 7.2 & 0.8 & 0.10 & 24.8 \\
$40-2,0-1$ & 5.2 & 0.5 & 0.03 & 24.8 \\
$40-2,3-4$ & 6.8 & 1.0 & 0.05 & 28.3 \\
$42-2,105-107$ & 1.7 & 0.5 & 0.25 & 34.0 \\
$44-3,23.5-24.5$ & 4.1 & 0.6 & 0.04 & 25.6 \\
$47-4,47-50$ & 6.4 & 0.5 & 0.03 & 20.0 \\
$48-3,123-124$ & 2.8 & 0.3 & 0.27 & 56.0 \\
$49-3,56-62$ & 5.3 & 0.5 & 0.01 & 26.5 \\
$49-3,90-93$ & 4.7 & 0.8 & 0.23 & 24.7 \\
$60-5,140-141$ & 5.0 & 0.8 & 0.04 & 22.7 \\
$63-4,19-23$ & 1.8 & 12.7 & 3.32 & 13.8 \\
$64-2,47-48$ & 4.6 & 2.0 & 0.01 & 27.1 \\
$64-2,79-80$ & 1.6 & 12.8 & 7.10 & 20.0 \\
$64-3,22-23$ & 3.7 & 1.3 & 0.01 & 16.8 \\
$64-3,24-25$ & 3.9 & 2.1 & 0.01 & 19.5 \\
$65-3,104-105$ & 4.6 & 2.1 & 0.01 & 18.4 \\
& & & & \\
Site 387 & & & & \\
$23-4,120-122$ & 8.2 & 0.5 & 0.03 & 15.6 \\
$25-3,144-146$ & 3.2 & 0.5 & 0.01 & 21.3 \\
$26-2,0-2$ & 6.1 & 0.7 & 0.01 & 19.7 \\
$27-1,39-41$ & 7.8 & 0.4 & 0.01 & 26.0 \\
$27-6,40-42$ & 4.8 & 0.3 & 0.02 & 20.0 \\
$28-1,131-133$ & 6.8 & 0.4 & 0.03 & 32.4 \\
$29-2,12-15$ & 11.5 & 0.5 & 0.01 & 24.0 \\
$29-4,101-106$ & 12.2 & 0.4 & 0.04 & 30.5 \\
$30-1,134-140$ & 2.5 & 1.2 & 0.01 & 20.8 \\
$35-5,0-2$ & 6.8 & 0.4 & 0.01 & 21.2 \\
$37-2,101-103$ & 5.1 & 0.6 & 0.01 & 23.2 \\
$42-1,72-75$ & 2.4 & 1.0 & 0.01 & 18.5 \\
$50-1,35-38$ & 8.3 & 0.6 & 0.01 & 13.4 \\
\hline & & & &
\end{tabular}

tion 1; 0-6 cm) and 385 (Cores 14 and 15). The $\mathrm{Fe} / \mathrm{Al}$ ratio in the red claystones of Site 386 is only slightly higher ( 0.5 to 1.0$)$; no high values were noted in the red claystones of Site 387. High values of the ratio characterize the lower portion of the Site 386 black and greenish gray claystone unit (Cores 49 to 65).

The $\mathrm{Mn} / \mathrm{Al}$ ratio is low $(0.01-0.03)$ throughout the sections drilled at Sites 382 and 385, except in several Mn-rich samples from the volcanogenic sequence, where values up to 0.1-0.2 were found. In the red claystones of Sites 386 and 387 , the $\mathrm{Mn} / \mathrm{Al}$ ratio does not exceed 0.1 , whereas in the black and gray claystone at Site 386 it is as high as 3.3 to 7.1 in some Mn-rich interbeds.

The $\mathrm{Al} / \mathrm{Ti}$ ratio in terrigenous sediments of Sites 382 and 385 ranges from 15.6 to 23.5. Some higher values were found in the pelagic sequence of Site 385, as well as in the upper portions of the volcanogenic sequence at both sites. No low-aluminum sediments were found in these intervals. The $\mathrm{Al} / \mathrm{Ti}$ ratio decreases sharply in the true volcaniclastic sediments, mostly owing to high titanium. It ranges from 1.4 to 17.1. Lowest values were found in the volcaniclastic breccia of Site 385 and in the volcaniclastic (heavy mineral) sandstone of Site 386. The Al/ Ti ratio in the black and greenish gray claystones at Site 386 is high, but tends to decrease from Core 49 downward. 


\section{Trace Elements}

$\mathrm{Zn}, \mathrm{Cr}, \mathrm{Cu}$, and $\mathrm{Ni}$ were determined in selected samples from most lithologic units drilled during Leg 43. Fewer determinations of Co were made, and these were mostly from Mesozoic rocks (Table 5). The examined trace elements are known to show different geochemical behavior during oceanic sedimentation and diagenesis. Concentrations of $\mathrm{Cu}, \mathrm{Ni}$, and $\mathrm{Co}$ increase in pelagic as compared with hemipelagic sediments; even richer in these elements are the active ridge "metalliferous" sediments (Boström et al., 1969; Skornyakova, 1976). In manganese nodules and Recent pelagic sediments $\mathrm{Ni}$ and $\mathrm{Cu}$ correlate with manganese, whereas $\mathrm{Co}$ is related to $\mathrm{Fe}$ oxides. Less certain are distribution patterns of $\mathrm{Zn}$; very high concentrations were found in the sulfide facies of the Red Sea hot brine deposits (Bischoff, 1969), but only a weak increase is noted in the pelagic and active ridge "metalliferous" sediments. Chromium is usually considered as being inert during sedimentation and diagenesis. It is mostly associated with the solid phases of sediments, especially with basaltic volcaniclastic matter. Low concentrations were found in the East Pacific Rise metalliferous sediments (Boström et al., 1969). However, soluble compounds of 6-valent $\mathrm{Cr}$ are known in highly oxidizing conditions of weathering and its possible mobility in these conditions should be taken into account.

No high anomalies of any examined trace element were found in the Leg 43 sediments. The ranges of concentration measured in terrigenous hemipelagic clay and silty clay at Sites 382 (Cores 1 through 15) and 385 (Cores 1 through 3 ) are only slightly exceeded in the Upper Cretaceous variegated claystones, volcaniclastic sediments, and black claystones. Most significant increases occur in the black claystones of Site 386.

Observing the trace elements distribution in different lithologies (Figures 1 through 5) the following regularities may be inferred. Almost parallel trends of $\mathrm{Cu}, \mathrm{Ni}$, $\mathrm{Zn}$, and $\mathrm{Cr}$ in the Pleistocene to Pliocene terrigenous turbidites at Site 382 (Figure 1), corresponding with $\mathrm{Fe}$ and $\mathrm{Fe} / \mathrm{Ti}$ curves, may be interpreted as an effect of dilution by detrital quartz and feldspar. On this background minor differences in individual distribution patterns of each element are almost invisible. Miocene hemipelagic clay at Sites 382 and 385 (Figures 1, 2) shows homogeneous and similar concentrations of all trace elements that are equivalent to the highest values found in turbidites. On the homogeneous background some extreme $\mathrm{Cu}$ (up to $280 \mathrm{ppm}$ ) and $\mathrm{Ni}$ (up to 416 ppm) appear. $\mathrm{Ni}$ in these cases is correlated with high manganese, the enrichment being likely caused by diagenetic concentration of both elements.

In the uppermost part of the Upper Cretaceous variegated sequences at Sites 382 (Cores 15, 16) and 385 (Cores 14, 15), trace elements show sharp variations. The trends are both subparallel and opposite. Concentrations of all trace elements are somewhat higher than those in hemipelagic clay, but high and low values do not coincide with color banding. Maxima of $\mathrm{Cu}$ (146 ppm and $150 \mathrm{ppm}$ ) were determined in Hole 382 ,
TABLE 5

Trace Elements in Leg 43 Sediments (ppm)

\begin{tabular}{|c|c|c|c|c|c|}
\hline $\begin{array}{c}\text { Sample } \\
\text { (Interval in } \mathrm{cm} \text { ) }\end{array}$ & $\mathrm{Zn}$ & $\mathrm{Cr}$ & $\mathrm{Cu}$ & $\mathrm{Ni}$ & Co \\
\hline \multicolumn{6}{|l|}{ Site 382} \\
\hline $1-1,59-61$ & 77 & 89 & 30 & 47 & - \\
\hline $1-2,60-62$ & 75 & 68 & 27 & 44 & - \\
\hline $1-3,126-128$ & 35 & 32 & 14 & 21 & - \\
\hline $1-4,90-92$ & 44 & 42 & 19 & 31 & - \\
\hline $1-4,113-115$ & 52 & 40 & 16 & 25 & 5 \\
\hline $1-4,127-129$ & 90 & 102 & 28 & 54 & - \\
\hline $1-5,110-112$ & 81 & 98 & 29 & 67 & - \\
\hline $1-6,40-42$ & 59 & 46 & 20 & 43 & - \\
\hline $2-1,60-70$ & 42 & 40 & 19 & 33 & - \\
\hline $2-2,90-92$ & 30 & 44 & 9 & 30 & - \\
\hline $2-3,48-50$ & 71 & 69 & 24 & 46 & - \\
\hline $2-3,64-66$ & 91 & 83 & 28 & 54 & - \\
\hline $2-3,92-94$ & 44 & 40 & 15 & 29 & - \\
\hline $3-1,126-128$ & 127 & 116 & 43 & 66 & - \\
\hline $3-2,35-37$ & 56 & 61 & 20 & 47 & - \\
\hline $3-2,100-102$ & 99 & 100 & 31 & 58 & - \\
\hline $3-2,147-149$ & 51 & 40 & 24 & 28 & - \\
\hline $4-1,80-82$ & 97 & 99 & 33 & 65 & - \\
\hline $5-1,96-98$ & 90 & 104 & 34 & 59 & - \\
\hline $5-2,95-97$ & 109 & 104 & 55 & 52 & - \\
\hline $5-3,100-102$ & 106 & 84 & 31 & 49 & - \\
\hline $6-1,53-55$ & 116 & 83 & 53 & 73 & - \\
\hline $6-1,148-150$ & 104 & 89 & 99 & 54 & - \\
\hline $7-2,81-83$ & 106 & 86 & 35 & 72 & 22 \\
\hline $7-6,67-69$ & 142 & 73 & 73 & 416 & - \\
\hline $8-3,108-110$ & 122 & 85 & 67 & 53 & 83 \\
\hline $8-5,97-99$ & 104 & 85 & 53 & 53 & - \\
\hline $9-1,100-102$ & 117 & 76 & 71 & 61 & - \\
\hline $9-5,120-122$ & 119 & 86 & 54 & 57 & - \\
\hline $10-2,4-7$ & 96 & 88 & 241 & 51 & - \\
\hline $11-1,96-98$ & 91 & 87 & 31 & 46 & - \\
\hline $11-4,76-78$ & 103 & 88 & 33 & 70 & - \\
\hline $12-2,90-92$ & 92 & 81 & 40 & 51 & - \\
\hline $12-4,103-105$ & 115 & 80 & 63 & 83 & - \\
\hline $13-1,34-36$ & 93 & 73 & 36 & 62 & - \\
\hline $13-4,59-61$ & 122 & 73 & 280 & 74 & - \\
\hline $14-1,36-38$ & 106 & 78 & 78 & 67 & 26 \\
\hline $14-5,123-125$ & 120 & 76 & 77 & 64 & - \\
\hline $15-5,10-12$ & 156 & 80 & 100 & 113 & 25 \\
\hline $15-6,44-47$ & 150 & 118 & 146 & 83 & 17 \\
\hline $15-6,44-47$ & 150 & 120 & 155 & 82 & 14 \\
\hline $15-6,60-62$ & 148 & 120 & 160 & 86 & 18 \\
\hline $16-1,0-3$ & 148 & 85 & 50 & 152 & 68 \\
\hline $16-1,94-98$ & 114 & 75 & 80 & 134 & 52 \\
\hline $16-4,99-105$ & 130 & 115 & 60 & 58 & 58 \\
\hline $16-6,45-48$ & 215 & 200 & 96 & 102 & 40 \\
\hline $16-6,123-126$ & 106 & 70 & 70 & 80 & 38 \\
\hline $17-1,102-104$ & 92 & 78 & 70 & 45 & 41 \\
\hline $17-4,115-119$ & 98 & 78 & 51 & 65 & 50 \\
\hline $17-5,79-81$ & 92 & 50 & 31 & 38 & 34 \\
\hline $18-1,61-65$ & 102 & 40 & 46 & 72 & 36 \\
\hline $18-2,4-9$ & 65 & 45 & 37 & 65 & 24 \\
\hline $18-2,109-112$ & 82 & 42 & 35 & 50 & 30 \\
\hline $18-4,9-12$ & 90 & 80 & 63 & 53 & 34 \\
\hline $19-4,20-25$ & 92 & 70 & 52 & 53 & 33 \\
\hline $20-2,36-38$ & 97 & 50 & 35 & 43 & 28 \\
\hline $20-5,55-57$ & 86 & 72 & 60 & 50 & 38 \\
\hline $21-3,82-84$ & 124 & 80 & 42 & 50 & 43 \\
\hline $22-2,120-122$ & 80 & 40 & 33 & 45 & 42 \\
\hline $22-3,148-150$ & 94 & 60 & 37 & 56 & 54 \\
\hline $23-2,33-35$ & 90 & 140 & 15 & 60 & 43 \\
\hline $24-1,90-93$ & 70 & 172 & 46 & 210 & 42 \\
\hline $25, \mathrm{CC}$ & 56 & 180 & 26 & 130 & 42 \\
\hline
\end{tabular}


TABLE 5 - Continued

\begin{tabular}{lrrrrr}
\hline $\begin{array}{c}\text { Sample } \\
\text { (Interval in cm) }\end{array}$ & Zn & Cr & $\mathrm{Cu}$ & $\mathrm{Ni}$ & $\mathrm{Co}$ \\
\hline Site 384 & & & & & \\
& & & & & \\
1-2, 28-30 & 20 & 24 & 52 & 34 & - \\
$2-2,90-92$ & 22 & 22 & 39 & 34 & - \\
$2-5,118-120$ & 61 & 37 & 45 & 30 & - \\
$3-1,110-112$ & 48 & 40 & 52 & 46 & - \\
$4-3,88-90$ & 54 & 88 & 46 & 34 & - \\
$4-5,122-124$ & 50 & 28 & 49 & 33 & - \\
$4-6,114-115$ & 68 & 20 & 238 & 83 & 310 \\
$5-1,100-102$ & 28 & 14 & 27 & 8 & - \\
$6-2,117-120$ & 44 & 18 & 52 & 32 & - \\
$7-3,16-18$ & 22 & 42 & 32 & 34 & - \\
$8-2,83-85$ & 32 & 28 & 56 & 36 & - \\
$9-3,37-39$ & 45 & 23 & 62 & 29 & - \\
$10-6,30-32$ & 46 & 29 & 43 & 42 & - \\
$11-2,82-84$ & 23 & 40 & 27 & 26 & - \\
$11-4,97-99$ & 50 & 38 & 34 & 30 & - \\
$12-5,117-119$ & 30 & 31 & 30 & 26 & - \\
$13-2,86-88$ & 23 & 20 & 17 & 27 & - \\
$13-5,88-90$ & 22 & 18 & 17 & 21 & - \\
$14-2,92-94$ & 11 & 19 & 14 & 18 & - \\
$15-2,15-17$ & 21 & 18 & 24 & 44 & - \\
22, CC brown & 80 & 530 & 69 & 210 & 85 \\
22, CC black & 85 & 430 & 69 & 140 & 70 \\
& & & & &
\end{tabular}

Site 385

$\begin{array}{lrrrrc}1-4,30-32 & 95 & 84 & 76 & 46 & - \\ 2-5,60-62 & 87 & 76 & 96 & 69 & - \\ 3-2,130-132 & 120 & 68 & 127 & 90 & 36 \\ 3-5,120-122 & 118 & 71 & 124 & - & - \\ 4-2,50-52 & 64 & 71 & 42 & 39 & - \\ 5-4,100-102 & 78 & 47 & 100 & 88 & - \\ 8-5,130-132 & 98 & 50 & 162 & 104 & 30 \\ 9-1,103-105 & 94 & 56 & 152 & 80 & - \\ 10-1,130-132 & 83 & 82 & 38 & 61 & - \\ 11-2,16-18 & 116 & 119 & 33 & 65 & - \\ 12-2,15-17 & 65 & 85 & 38 & 53 & - \\ 12-2,31-33 & 87 & 100 & 39 & 100 & 30 \\ 13-2,30-39 & 98 & 100 & 41 & 80 & 38 \\ 13-3,109-111 & 85 & 108 & 35 & 52 & 34 \\ 13-4,144-147 & 85 & 120 & 39 & 74 & 46 \\ 14-1,68-70 & 117 & 85 & 73 & 106 & 34 \\ 14-2,6-8 & 118 & 132 & 72 & 74 & 50 \\ 15-1,53-55 & 140 & 90 & 126 & 140 & 46 \\ 15-1,100-101 & 120 & 82 & 105 & 135 & 75 \\ 15-1,148-150 & 120 & 85 & 104 & 146 & 105 \\ 16-2,8-10 & 152 & 92 & 96 & 82 & 34 \\ 16-3,77-80 & 148 & 85 & 90 & 160 & 92 \\ 20-2,40-50 & 89 & 125 & 48 & 122 & 44 \\ 23-1,128-134 & 112 & 310 & 69 & 118 & 74 \\ 23-3,142-150 & 126 & 220 & 55 & 96 & 54\end{array}$

\section{Site 386}

\begin{tabular}{lrrrrr}
$4-2,111-115$ & 138 & 138 & 77 & 139 & - \\
$4-4,124-129$ & 168 & 98 & 58 & 80 & - \\
$5-2,46-50$ & 150 & 148 & 72 & 116 & - \\
$6-4,128-133$ & 88 & 150 & 82 & 83 & - \\
$8-1,147-150$ & 104 & 60 & 82 & 65 & - \\
$12-4,8-10$ & 111 & 148 & 82 & 110 & - \\
$13-3,127-133$ & 90 & 200 & 60 & 113 & 58 \\
$14-1,1-5$ & 68 & 62 & 35 & 18 & - \\
$14-4,19-21$ & 134 & 132 & 78 & 80 & 50 \\
$15-2,81-85$ & 41 & 64 & 23 & 21 & - \\
$.17-1,132-135$ & 22 & 22 & 12 & 10 & - \\
$18-2,37-39$ & 39 & 40 & 29 & 22 & - \\
$34-2,25-27$ & 62 & 110 & 25 & 31 & 7 \\
\hline
\end{tabular}

TABLE 5 - Continued

\begin{tabular}{lrrrrr}
\hline \multicolumn{1}{c}{$\begin{array}{c}\text { Sample } \\
\text { (Interval in cm) }\end{array}$} & $\mathrm{Zn}$ & $\mathrm{Cr}$ & $\mathrm{Cu}$ & $\mathrm{Ni}$ & $\mathrm{Co}$ \\
\hline $36-5,74-76$ & 148 & 86 & 96 & 156 & 85 \\
$36-5,94-98$ & 112 & 130 & 57 & 120 & 85 \\
$38-1,26-32$ & 75 & 70 & 76 & 66 & 50 \\
$38-2,145-146$ & 82 & 46 & 70 & 95 & 38 \\
$38-2,149-150$ & 90 & 48 & 67 & 73 & 30 \\
$38-3,90-91$ & 54 & 35 & 45 & 46 & 37 \\
$39-1,129-131$ & 100 & 56 & 75 & 82 & 52 \\
$39-1,144-145$ & 100 & 64 & 120 & 97 & 70 \\
$40-2,0-1$ & 78 & 70 & 41 & 51 & 34 \\
$40-2,3-4$ & 90 & 50 & 45 & 112 & 58 \\
$45-5,84-88$ & 78 & 44 & 67 & 68 & 20 \\
$47-4,47-50$ & 140 & 54 & 110 & 180 & 78 \\
$49-3,56-62$ & 67 & 44 & 56 & 80 & 5 \\
$50-3,35-36$ & 100 & 20 & 60 & 410 & 180 \\
$51-2,68-69$ & 65 & 84 & 71 & 286 & 132 \\
$53-1,120-121$ & 58 & 48 & 30 & 170 & 86 \\
$54-4,100-101$ & 116 & 96 & 78 & 165 & 72 \\
$55-2,59-60$ & 86 & 20 & 14 & 76 & 34 \\
$56-5,143-144$ & 78 & 48 & 62 & 116 & 28 \\
$57-6,23-26$ & 160 & 56 & 66 & 246 & 36 \\
$59-4,57-60$ & 275 & 66 & 125 & 310 & 72 \\
$60-5,140-141$ & 220 & 94 & 134 & 110 & 50 \\
$64-2,47-48$ & 160 & 40 & 58 & 100 & 6 \\
$64-3,22-23$ & 80 & 82 & 34 & 75 & 38 \\
$64-3,24-25$ & 70 & 35 & 75 & 80 & 24 \\
$65-3,104-105$ & 135 & 196 & 80 & 245 & 36
\end{tabular}

Site 387

\begin{tabular}{|c|c|c|c|c|c|}
\hline $1-6,80-82$ & 111 & 91 & 80 & 77 & - \\
\hline $2-2,80-82$ & 107 & 96 & 118 & 64 & - \\
\hline $3-2,130-132$ & 121 & 86 & 81 & 63 & - \\
\hline $6-3,70-73$ & 110 & 81 & 96 & 70 & - \\
\hline $7-3,80-83$ & 50 & 64 & 123 & 37 & - \\
\hline $10-1,146-150$ & 24 & 52 & 52 & 64 & - \\
\hline $13-1,104-106$ & 75 & 91 & 40 & 40 & - \\
\hline $16-3,58-60$ & 35 & 40 & 40 & 45 & 16 \\
\hline $23-4,120-122$ & 90 & 66 & 70 & 65 & 20 \\
\hline $25-3,144-146$ & 47 & 100 & 21 & 6 & 10 \\
\hline $27-1,39-41$ & 108 & 135 & 50 & 64 & 16 \\
\hline $27-6,40-42$ & 53 & 88 & 40 & 60 & 16 \\
\hline $28-1,131-133$ & 98 & 115 & 52 & 75 & 20 \\
\hline $29-2,12-15$ & 120 & 93 & 45 & 101 & 19 \\
\hline $29-4,101-106$ & 118 & 98 & 100 & 90 & 20 \\
\hline $30-1,134-140$ & 168 & 57 & 148 & 318 & 5 \\
\hline $33-2,40-45$ & 51 & 40 & 42 & 68 & 8 \\
\hline $34-2,103-106$ & 45 & 20 & 12 & 80 & 10 \\
\hline $35-5,0-2$ & 42 & 52 & 44 & 40 & 10 \\
\hline $36-1,71-73$ & 50 & 64 & 23 & 38 & 16 \\
\hline $37-3,101-103$ & 65 & 56 & 49 & 190 & 6 \\
\hline $40-2,52-54$ & 95 & 66 & 66 & 80 & 12 \\
\hline $42-1,72-75$ & 145 & 90 & 90 & 80 & 42 \\
\hline $44-1,71-75$ & 24 & 20 & 8 & 60 & 5 \\
\hline $46-1,86-88$ & 12 & 38 & 8 & 54 & 5 \\
\hline $48-1,119-121$ & 20 & 38 & 12 & 54 & 5 \\
\hline $49-5,45-50$ & 40 & 30 & 67 & 60 & 14 \\
\hline $50-1,35-38$ & 80 & 410 & 92 & 98 & 58 \\
\hline
\end{tabular}

Core 15 , Section 6 , together with relatively high $\mathrm{Zn}$ (150 ppm) and $\mathrm{Cr}(120 \mathrm{ppm})$, while $\mathrm{Ni}$ and Co show relative minima in the same samples. Peaks of $\mathrm{Cr}(200$ $\mathrm{ppm})$ and $\mathrm{Zn}(215 \mathrm{ppm})$ in Core 16, Section 6, 45-48 $\mathrm{cm}$, correspond with small peaks of $\mathrm{Cu}(96 \mathrm{ppm})$ and $\mathrm{Ni}(102 \mathrm{ppm})$ and with very high Fe (10 per cent); Co and Mn, however, do not show any increase. Trace element concentrations are probably controlled by differ- 


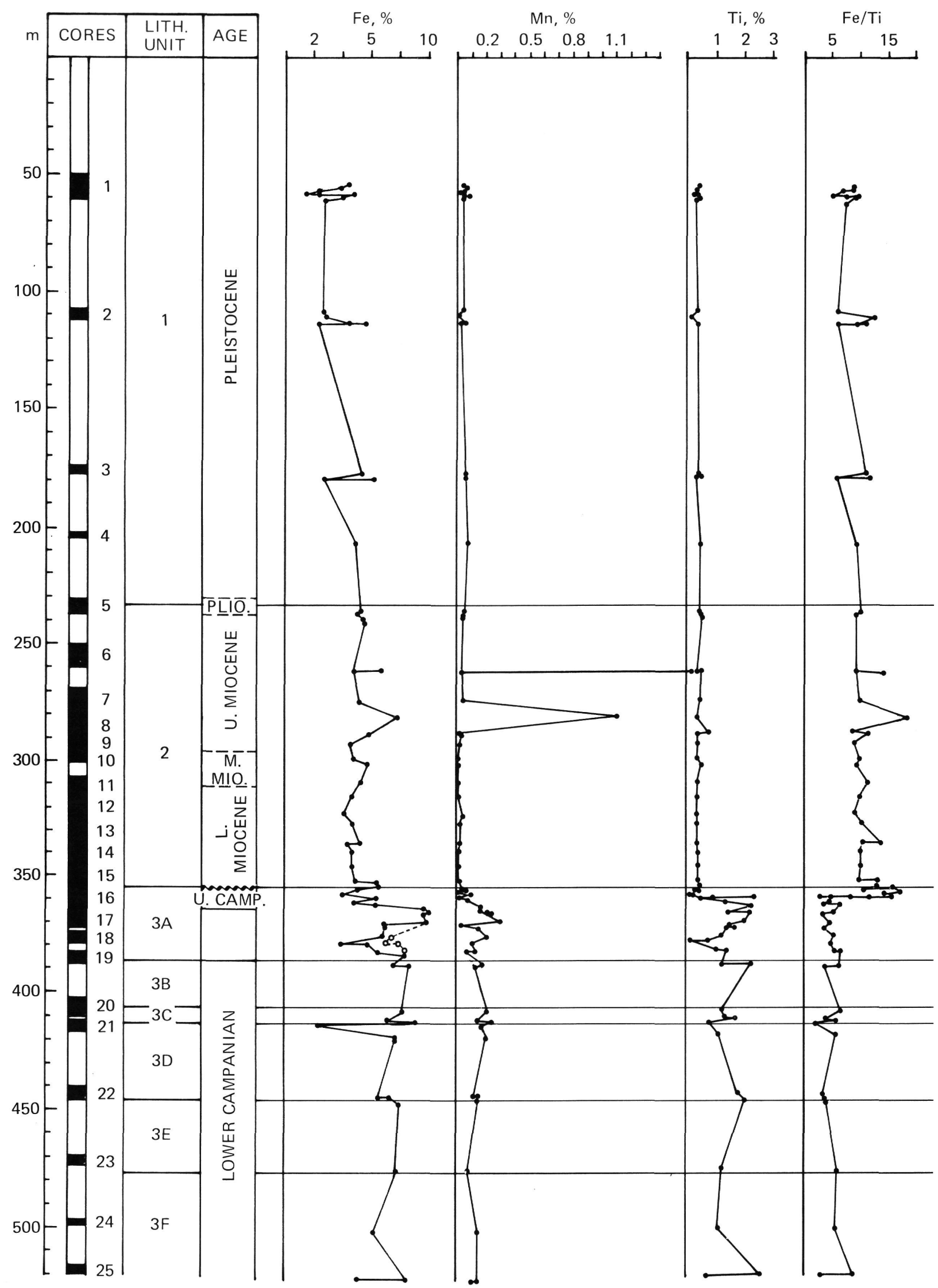

Figure 1. Geochemical data, Site 382. Dotted lines on the Fe and Mn graphs show the elements on a carbonatefree basis; other data are on the basis of bulk sample analyses. 
INORGANIC GEOCHEMISTRY

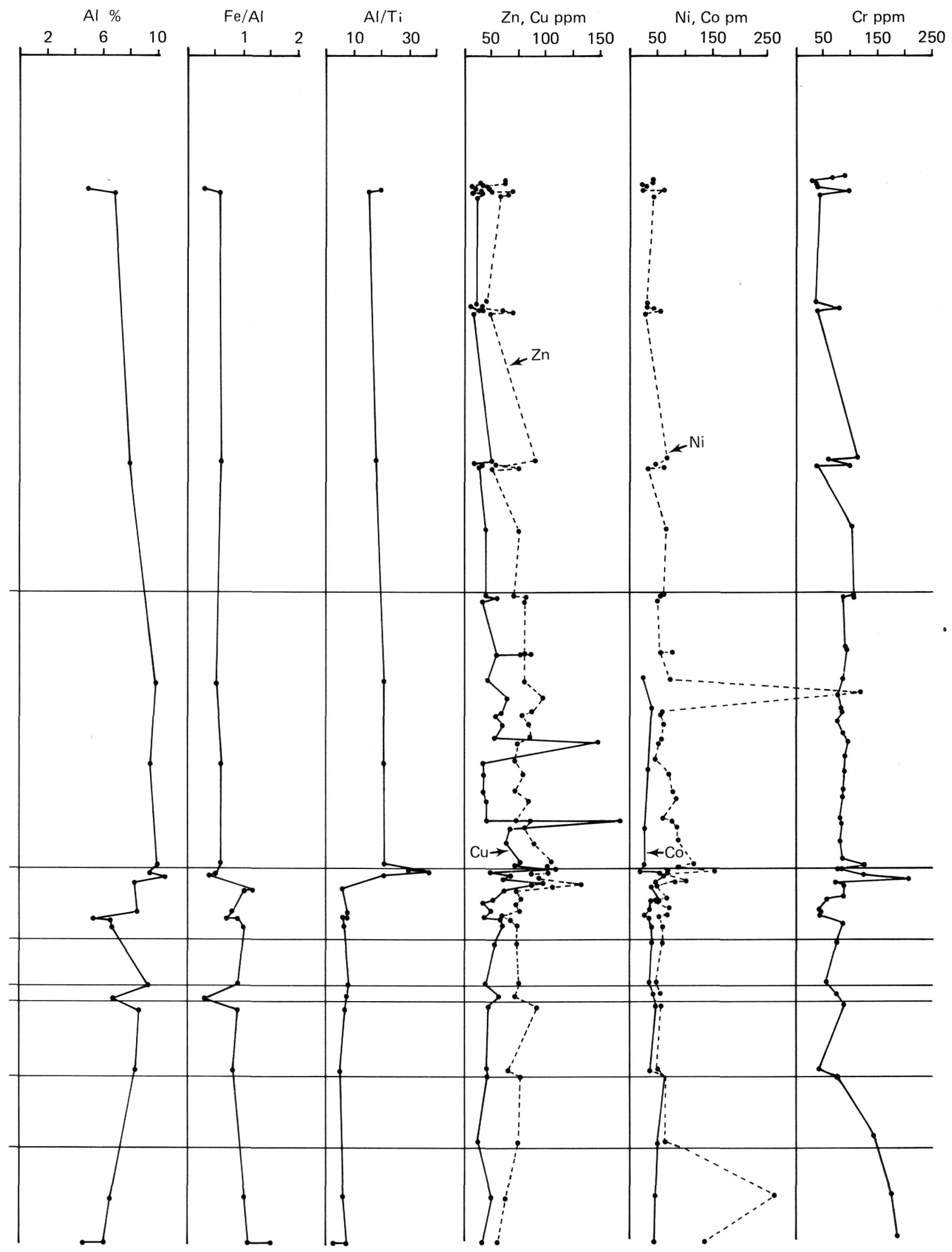

Figure 1. (Continued).

685 


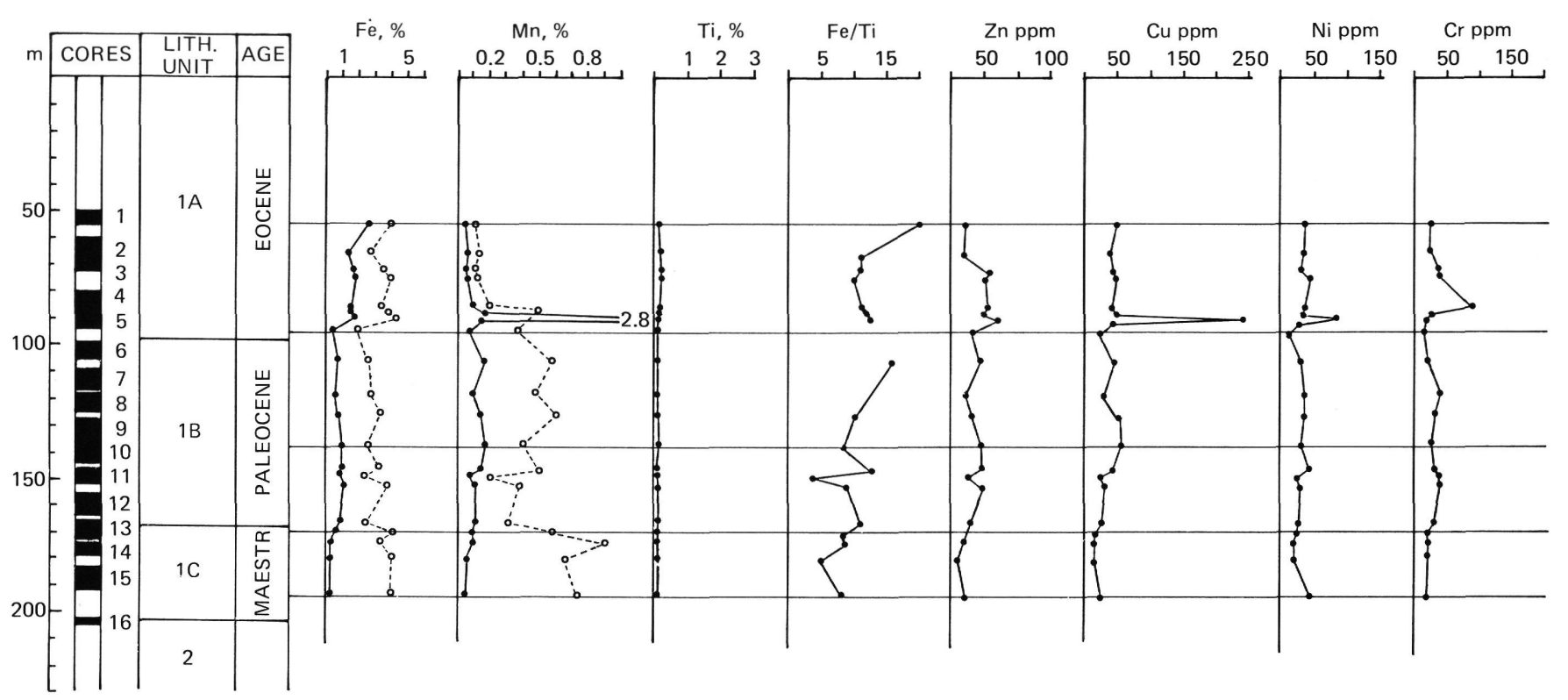

Figure 2. Geochemical data, Site 384. Dotted lines on the Fe and Mn graphs show the elements on a carbonate-free basis; other data are on the basis of bulk sample analyses.

ent overlapping factors. Among these factors, diagenetic migration in contrasting redox conditions seems to be important. But the relative abundance of all trace elements in most samples may be considered to be a result of their pelagic concentration.

It is somewhat puzzling that other portions of the volcanogenic sequences in Holes 382 and 385 show rather low values of $\mathrm{Ni}$ and $\mathrm{Cr}$, whereas those in volcaniclastic breccia and basalts are high. The volcanogenic clay and silt contain even less $\mathrm{Cr}$ (40 to $80 \mathrm{ppm}$ ) than hemipelagic clay ( 75 to $90 \mathrm{ppm}$ ). Leaching of $\mathrm{Cr}$ and $\mathrm{Ni}$ from primary basaltic volcaniclastics during their alteration is probably responsible for the low values.

The volcaniclastic turbidites of Hole 386 (Figure 4) are considerably enriched in $\mathrm{Cr}$ (up to $200 \mathrm{ppm}$ ), $\mathrm{Zn}$ (up to $150 \mathrm{ppm}$ ), and $\mathrm{Ni}$ (up to $139 \mathrm{ppm}$ ), whereas $\mathrm{Cu}$ and $\mathrm{Co}$ show normal or low values. $\mathrm{Cu}, \mathrm{Ni}$, and $\mathrm{Cr}$ are probably associated with volcaniclastic material and with its alteration products. There is no evidence of leaching.

The Eocene siliceous and calcareous turbidites at Sites 386 and 387 are commonly low in trace elements except for some samples that show a relatively parallel increase in $\mathrm{Zn}$ and $\mathrm{Cr}$, or an independent increase in $\mathrm{Cu}$ (Figures 4, 5).

The Upper Cretaceous variegated and red claystones at Sites 386 and 387 (Figures 4, 5) are slightly enriched in $\mathrm{Zn}$ (up to $148 \mathrm{ppm}$ ), less in $\mathrm{Cu}$ (up to 120 $\mathrm{ppm}$ ) and $\mathrm{Ni}$ (up to $156 \mathrm{ppm}$ ). These values, however, are lower than averages in Recent pelagic clay (Skornyakova, 1976).

Considerable variations in $\mathrm{Zn}, \mathrm{Cu}$, and $\mathrm{Ni}$ were found in the black and greenish gray claystones at Site 387 (Figure 5), whereas $\mathrm{Co}$ and $\mathrm{Cr}$ are uniformly low. In the lithologically similar sediments at Site 386 the variations are even more significant and involve Co
(Figure 4). The lower part of the black claystone sequence is enriched in $\mathrm{Zn}$ (up to $275 \mathrm{ppm}$ ); $\mathrm{Ni}$ (up to $310 \mathrm{ppm}$ ), and $\mathrm{Cu}$ (up to $134 \mathrm{ppm}$ ); high concentrations of $\mathrm{Ni}$ and $\mathrm{Co}$ were found in the upper portion. The trace elements, especially $\mathrm{Ni}$, tend to correlate with manganese. Influence of a mid-oceanic exhalative source is assumed to explain the increased concentrations of the trace elements in sediments directly overlying basaltic basement. However, extreme values are probably a result of diagenetic migration.

In the Lower Cretaceous limestones and calcareous claystones at Site 387 trace elements are diluted by carbonate (Figure 5); the same is apparent in the section of calcareous sediments at Site 384 (Figure 2).

\section{DISCUSSION}

The geochemical investigation of the Leg 43 samples is not yet complete and the present contribution should be taken as preliminary. It seems reasonable, therefore, to confine the present discussion to several geochemical problems that bear on the sedimentology of the Leg 43 sediments. These can be summarized as (1) the geochemistry of variegated and red claystones, (2) "metalliferous" trends in the black and greenish gray claystones, and (3) silicification.

\section{Variegated And Red Claystones}

Three different kinds of red-and brown-colored (oxidized) sediments were observed during Leg 43: (1) pelagic, comprising calcareous ooze at Site 384 , pelagic clay and radiolarian ooze or mud at Sites 385 (Unit 2) and 387 (Units 1,2); (2) volcanogenic sequences at Sites 382 and 385; (3) red claystones at Sites 386 (Unit 5 ) and 387 (Unit 5). The last two are discussed here.

The lowermost lithologic units at Sites 382 and 385 are interpreted as volcanogenic, reflecting volcanic activity on the New England seamounts. The interpreta- 


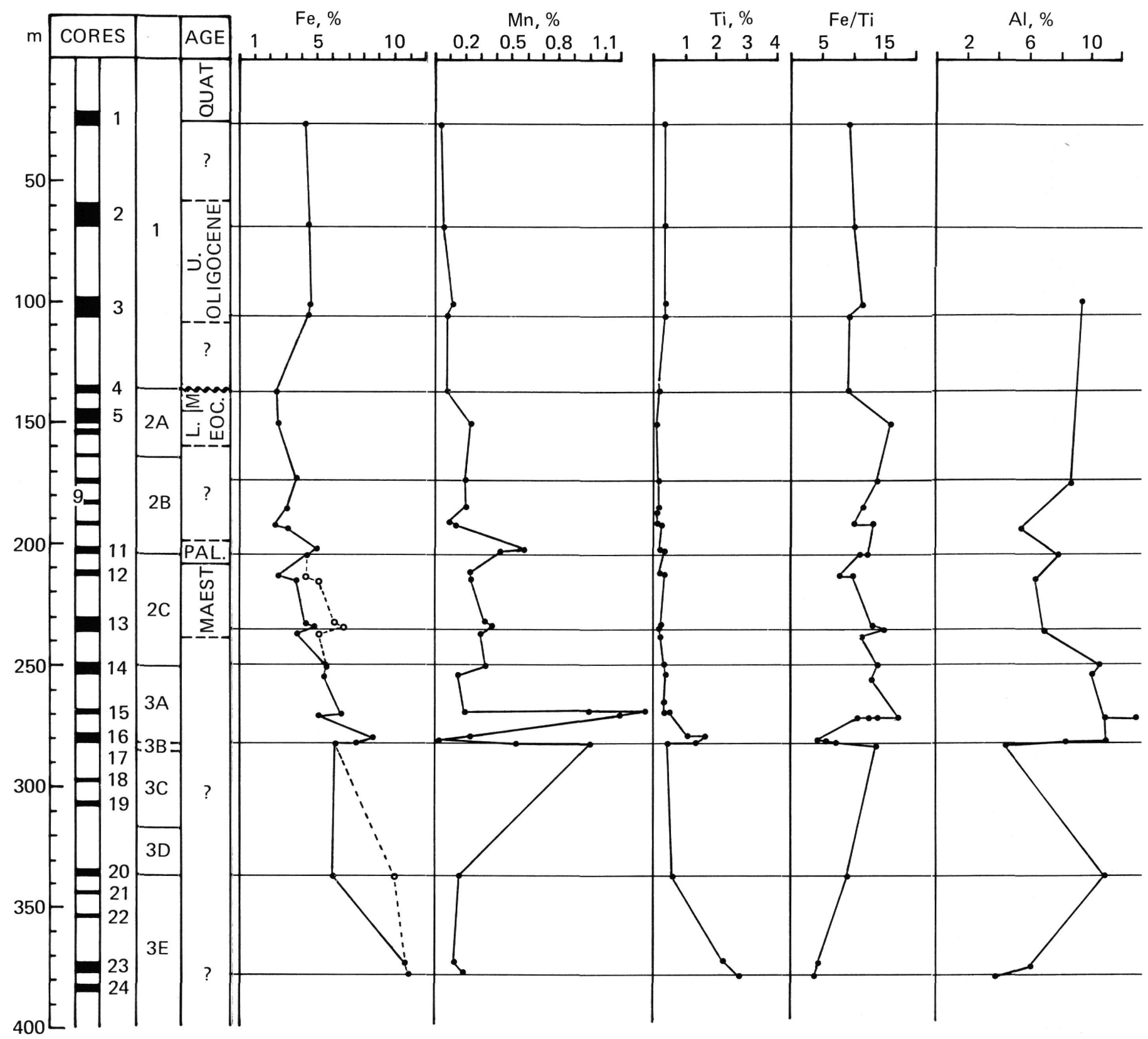

Figure 3. Geochemical data, Site 385. Dotted lines on the Fe and Mn graphs show the elements on a carbonate-free basis; other data are on the basis of bulk sample analyses.

tion is based on shipboard visual and smear-slide descriptions, and confirmed by shore-laboratory mineralogical analyses. However, a primary volcaniclastic origin is not evident in some fine-grained clay or silty clay layers in the upper portions of the units. These display significant geochemical differences, so that there are two geochemically different parts in each of the two units.

The lower, evidently volcaniclastic parts (Site 382 , Cores 16 to 25; Site 385 , Cores 16 to 23 ) are enriched both in $\mathrm{Fe}$ and $\mathrm{Ti}$, the latter being so high, that $\mathrm{Fe} / \mathrm{Ti}$ ratios, as well as ratios of $\mathrm{Al} / \mathrm{Ti}$ and $\mathrm{Mn} / \mathrm{Ti}$ appear to be very low. The titanium is obviously enclosed in Tibearing clastic minerals derived from primary basalts or from their alteration products. Presedimentary alteration of basalts, leading to relative concentration of $\mathrm{Ti}$ in the alteration products is necessary, because Ti content in some sediment layers exceeds that in basalt fragments from the volcaniclastic breccia. Abundant sphene, in some cases together with anatase, was found in the coarse heavy fraction of these sediments.

Most of the iron is also probably clastic in origin, being related to basic volcaniclastic matter, which itself is enriched in $\mathrm{Fe}$ during the course of alteration, as shown by the analyzed volcaniclastic breccia. The phase analyses show that insoluble $\mathrm{Fe}^{+3}$ dominates over the soluble form (amorphous or poorly crystallized hydroxides) in most samples. Relatively high per- 


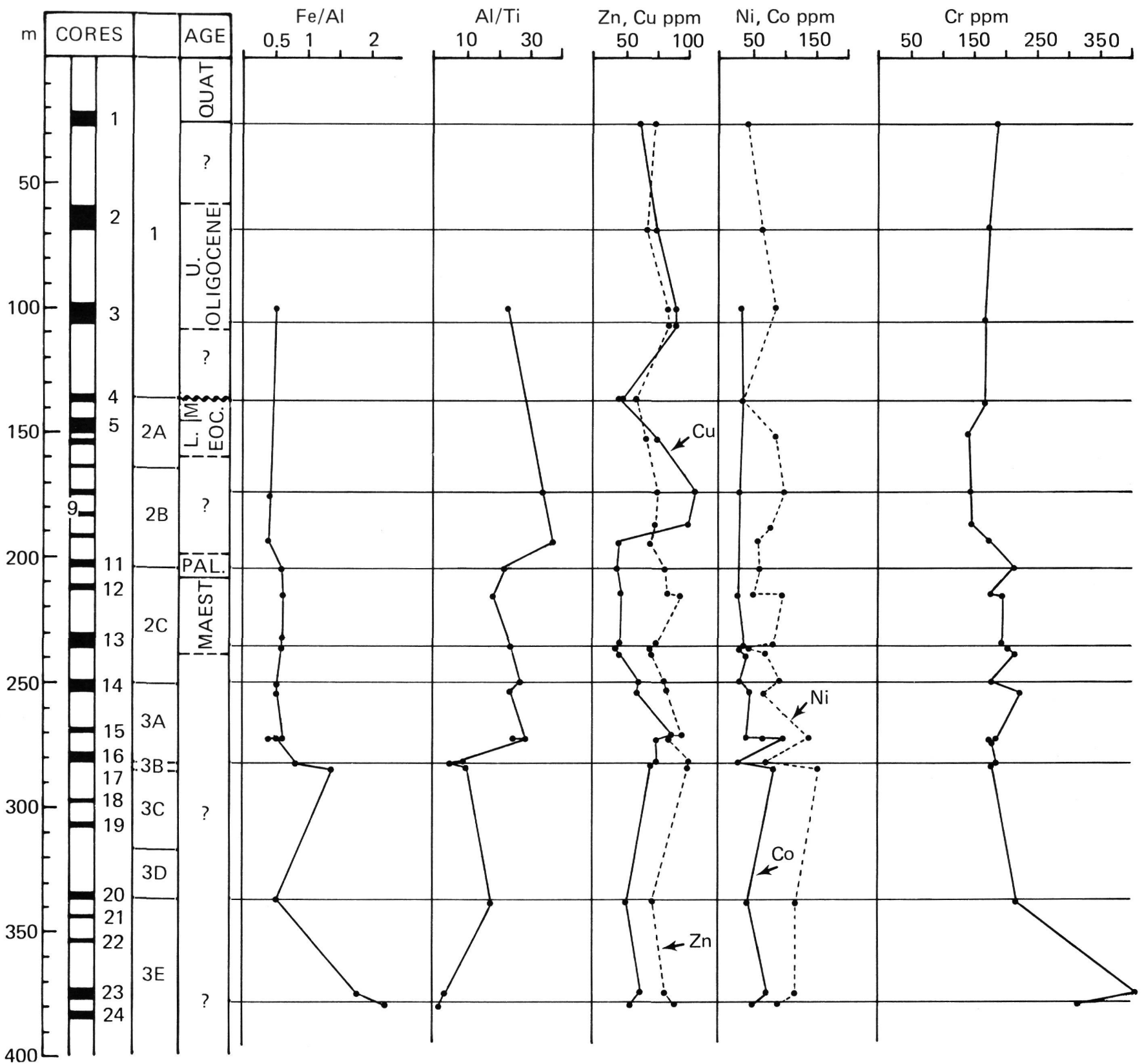

Figure 3. (Continued).

centages of the soluble $\mathrm{Fe}^{+3}$ are found, however, in both the sediments and the breccia. It seems likely, therefore, that dispersed iron hydroxides are in large part also of detrital origin rather than precipitated chemically in situ from seawater or from hydrothermal solutions. Moderate values of the $\mathrm{Fe} / \mathrm{Al}$ ratio confirm this assumption. Of course, precipitation is not excluded completely.

From the geochemical data it can be concluded that Ti- and Fe-rich clastic matter, both coarse and finegrained, was derived from altered basaltic volcanic rocks of the seamounts and deposited mechanically on their lower slopes. Minor post-depositional migration (hydrothermal or diagenetic) may result in the local additional concentration of iron hydroxide. No clear evidence of "metalliferous" sediments was found in the sequences described above.
The upper portions of the volcanogenic units show different geochemical patterns. The most significant seems to be low titanium content, which results in high $\mathrm{Fe} / \mathrm{Ti}, \mathrm{Al} / \mathrm{Ti}$, and $\mathrm{Mn} / \mathrm{Ti}$ ratios. On the other hand, $\mathrm{Al}$ is relatively high; thus, $\mathrm{Fe} / \mathrm{Al}$ and $\mathrm{Mn} / \mathrm{Al}$ are rather low in spite of high $\mathrm{Fe}$ and slightly enriched $\mathrm{Mn}$. The concentrations of $\mathrm{Fe}$ and $\mathrm{Mn}$, therefore, cannot be interpreted as "metalliferous." The layers are geochemically similar to pelagic, rather than to volcanogenic sediments. Relatively high $\mathrm{SiO}_{2}$ content and $\mathrm{SiO}_{2} /$ $\mathrm{Al}_{2} \mathrm{O}_{3}$ ratios, as compared with those in volcaniclastic sediments, confirm this conclusion.

The same patterns appear in the red claystones of Sites 386 (Unit 5) and 387 (Unit 5), which overlie black and greenish gray claystones. At Site 387 these are partly silicified resulting in lower metal concentrations. The ratios of the metals with $\mathrm{Ti}$ and $\mathrm{Al}$ tend to 


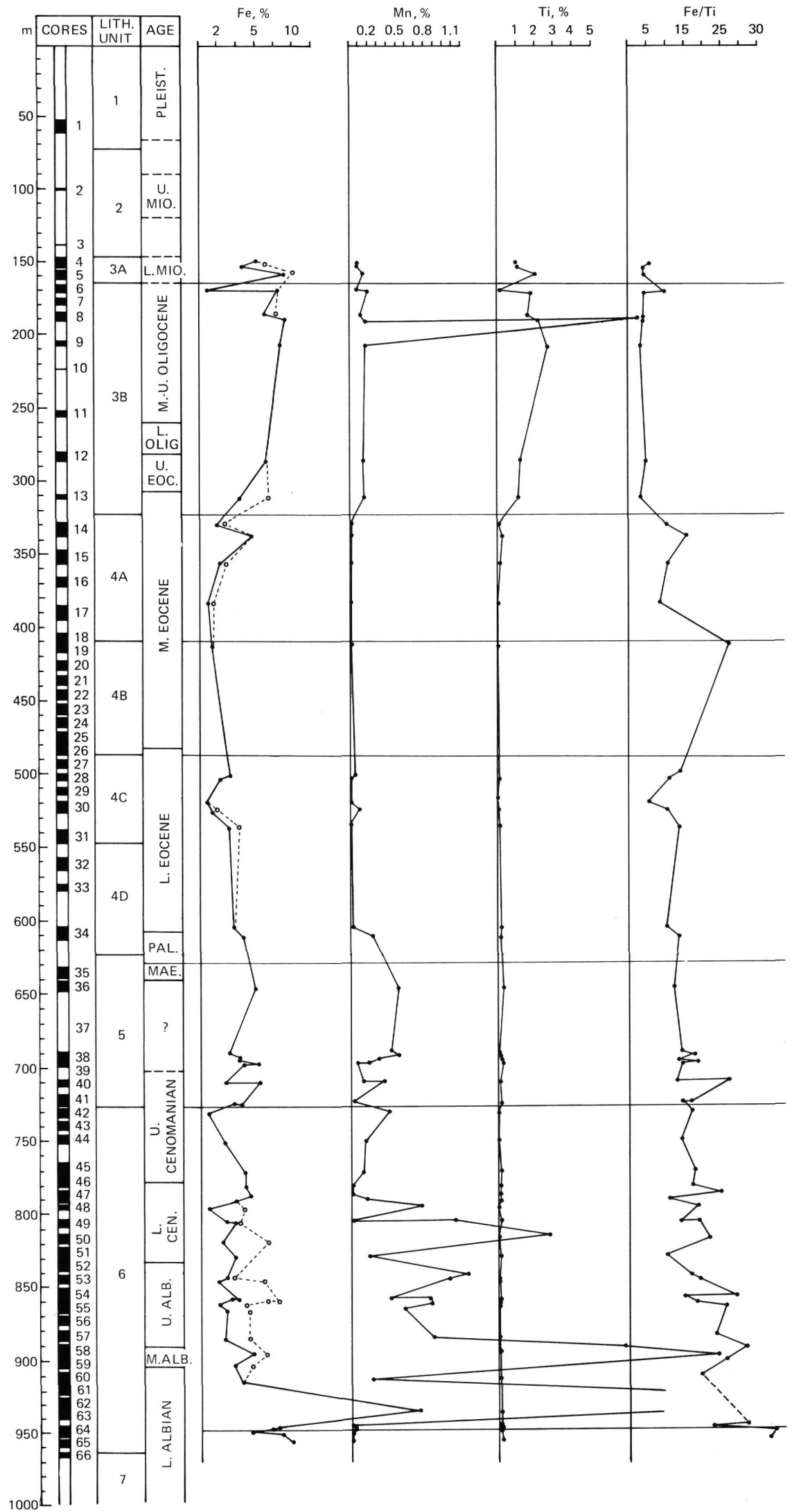

Figure 4. Geochemical data, Site 386. Dotted lines on the Fe and Mn graphs show the elements on a carbonate-free basis; other data are on the basis of bulk sample analyses. 
I. O. MURDMAA, V. V. GORDEEV, E. M. EMELYANOV, E. S. BAZILEVSKAYA

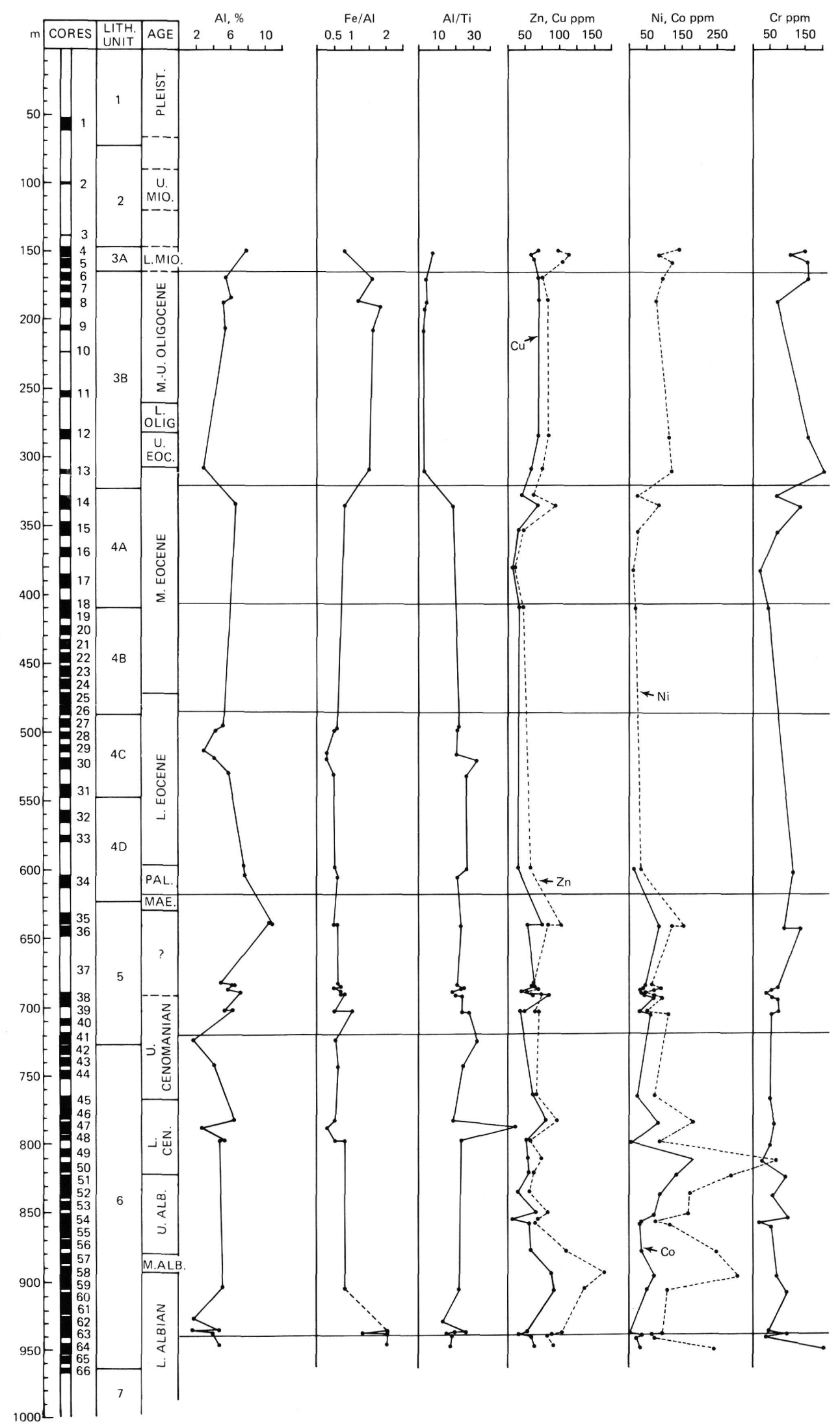

Figure 4. (Continued). 


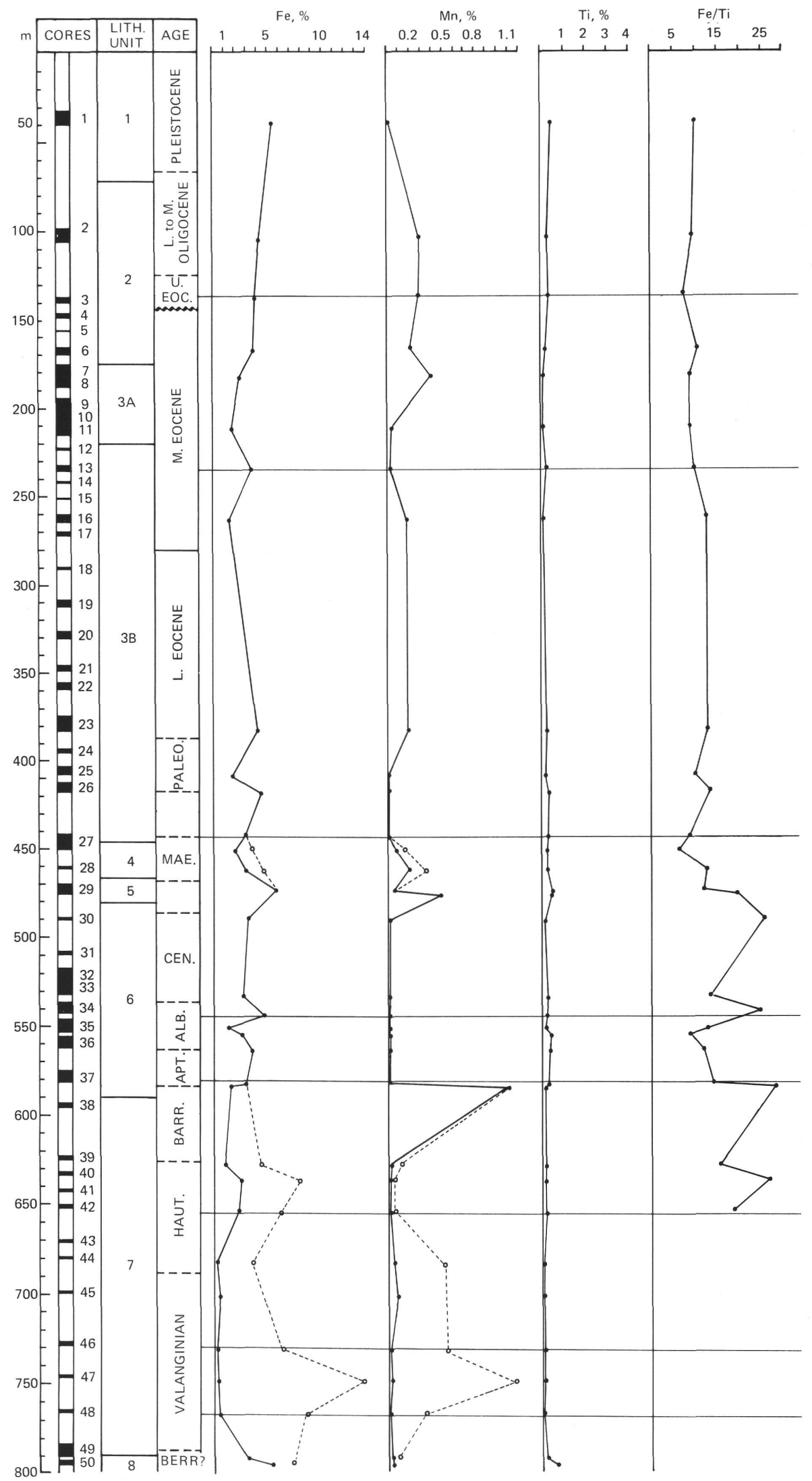

Figure 5. Geochemical data, Site 387. Dotted lines on the Fe and Mn graphs show the elements on a carbonate-free basis; other data are on the basis of bulk sample analyses. 
I. O. MURDMAA, V. V. GORDEEV, E. M. EMELYANOV, E. S. BAZILEVSKAYA

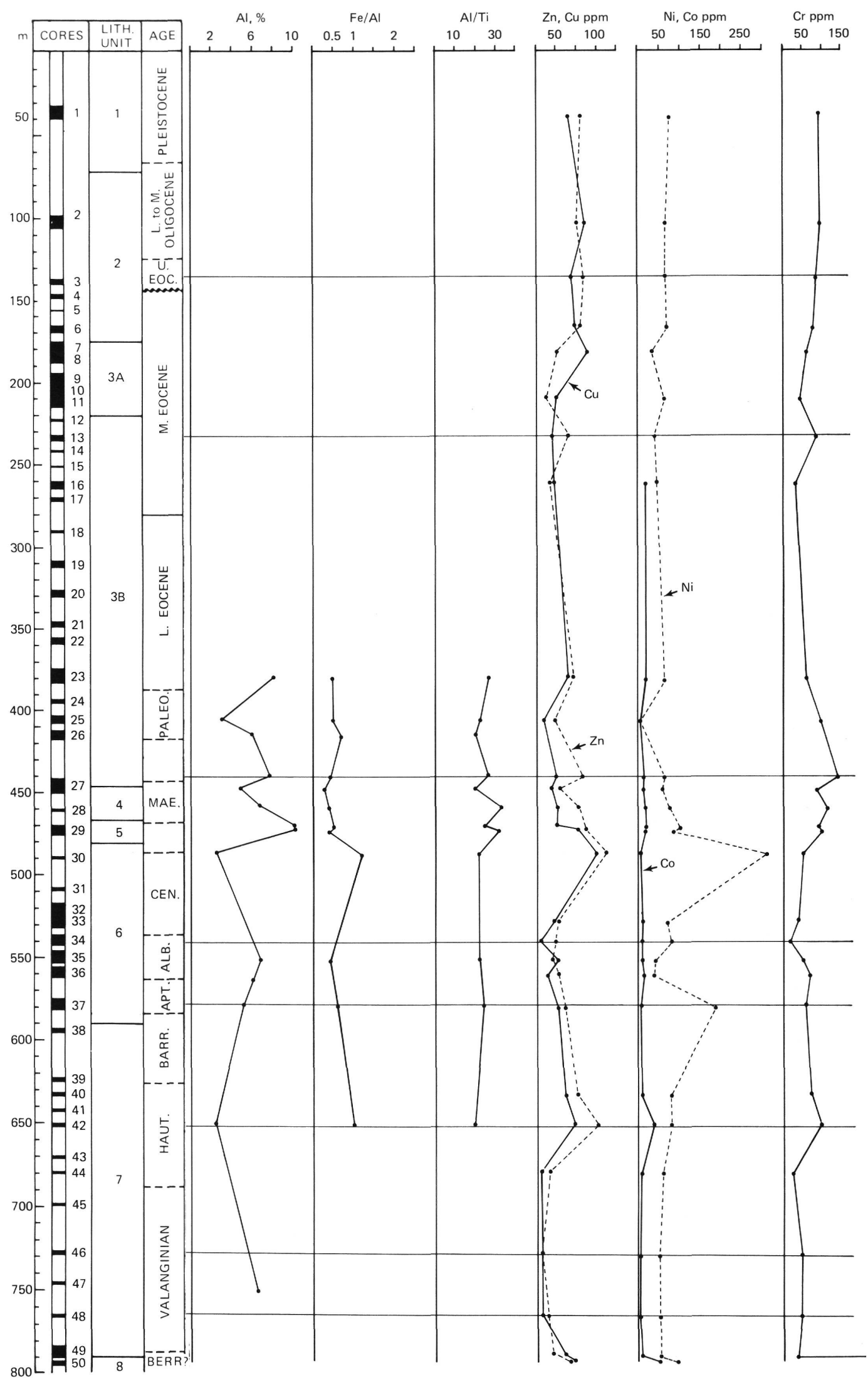

Figure 5. (Continued). 
be higher than in "normal" terrigenous clay. The relative enrichment in iron and manganese, however, is not sufficient to indicate that this is a "metalliferous" trend. It should be attributed rather to a pelagic environment and low rate of accumulation.

Thus, two kinds of relative concentration of $\mathrm{Fe}$ in oxidized sediments are indicated, the first related to basic volcaniclastic materials where $\mathrm{Fe}$ increases together with $\mathrm{Ti}$, the second, pelagic, where high $\mathrm{Fe}$ content appears to parallel increased or normal $\mathrm{Al}$ and results from low accumulation rates.

\section{"Metalliferous" Trends In The Lower Cretaceous Black And Greenish Gray Claystones}

Interbeds with high pyrite and/or siderite content occur sporadically throughout the carbon-rich claystone sequence. High concentrations of $\mathrm{Mn}$, resulting from authigenic rhodochrosite, are less common. Against the background of low concentrations, one can observe layers regularly enriched in the metals in the lower portion of Unit 6 at Site 387, which is underlain by basalt.

There are two intervals, an upper one rich in $\mathrm{Mn}$ (Cores 49 to 63) and a lower one rich in $\mathrm{Fe}$ (Cores 58 to 65) that together span a thickness of about 160 meters. Increased concentrations of these elements appear regularly in these intervals, but even more evident are the high $\mathrm{Fe} / \mathrm{Ti}, \mathrm{Fe} / \mathrm{Al}, \mathrm{Mn} / \mathrm{Ti}$, and, in a few analyzed samples, $\mathrm{Mn} / \mathrm{Al}$ ratios. The increased abundances of $\mathrm{Fe}$ and $\mathrm{Mn}$ versus geochemically inert $\mathrm{Ti}$ and $\mathrm{Al}$, indicate enrichment of both metals as compared with normal terrigenous matter. Excess $\mathrm{Fe}$ is found to be in siderite or manganic siderite as well as in pyrite (X-ray and phase analyses data). $\mathrm{Mn}$ is contained in rhodochrosite, manganic siderite, and/or manganic calcite. The minerals are undoubtedly of post-depositional (diagenetic?) origin, but the time and mode of formation of the high metal concentrations are not clear.

Two alternative hypotheses may be proposed. The first is precipitation from the interstitial water during diagenesis in active reducing conditions, produced by very high content of organic matter. Migrating in solution as reduced ions, the metals precipitate as sulfides and carbonates as a result of diagenetic reactions in carbonate-rich or sulfur-rich layers. If so, a source of $\mathrm{Fe}$ and $\mathrm{Mn}$ within the sediment column (or below) is necessary, sufficient to provide the great amounts of the metals now found in the thick sequence. It may be the underlying basalt, but no direct evidence of extensive leaching was found, at least in drilled basement samples.

Another possible explanation is an exhalation-sedimentation hypothesis, as proposed for the metalliferous sediments adjacent to mid-oceanic ridges. if the endogenic source of the metals is the ocean-floor spreading center, as it is assumed in the case of metalliferous sediments, then observed partial separation of $\mathrm{Fe}$ and $\mathrm{Mn}$ in the sediment column can be easily explained. Indeed, the lower (iron-rich) portion was then accumulated directly on the newly formed basaltic basement, i.e., close to the spreading ridge crest. Fe was precipitated from the solution first, forming high-iron sediments adjacent to the source area. Afterwards, when the site was sufficiently remote from the ridge crest, $\mathrm{Mn}$ was precipitated. A similar situation is proposed for the recent Mid-Pacific rise, where $\mathrm{Fe}$-rich metalliferous sediments tend to be closer to the ridge crest than Mnrich ones of the Bauer depression.

Even if this assumption is not correct, comparison of the $\mathrm{Fe}$ - and $\mathrm{Mn}$-rich layers at Site 386 with metalliferous sediments seems reasonable. In contrast with other known oceanic oxidized metalliferous sediments we have here an example of strongly reducing conditions.

Further investigations are necessary to resolve this problem.

\section{Silicification}

Bulk analyses revealed high $\mathrm{SiO}_{2}$ content in several intervals at Sites 386 and 387, where the rocks have been described as claystones, silicified claystones, or altered radiolarian mudstones. These silica-rich intervals comprise, at least partly, Unit 4 (only a few samples were analyzed), the whole of Unit 5, and most of Unit 6 at Site 386; and Units $3 \mathrm{~B}$ and 5 at Site 387. The transition from sediments with normal $\mathrm{SiO}_{2}$ to highly siliceous varieties is usually marked in the visual core descriptions by an increase in lithification. On the graphs of physical properties it coincides with increased density and decreased water content. X-ray data show abundant disordered cristobalite in the upper parts of the siliceous intervals (Eocene), whereas the lower parts are rich in quartz (Paleocene to Lower Cretaceous) (see Koch and Rothe, this volume). In the Eocene siliceous rocks abundant microspherules (lepispheres) were found by scanning electron microscopy, often associated with clinoptilolite crystals. Cristobalitic, low birefringence,poikiloblastic matrix is visible in thin sections. In "quartzose" varieties, rosettes of chalcedony are common, replacing radiolarians and occurring as poikiloblastic matrix.

Summarizing the data and comparing them with chemical features, one can assume that extensive silicification has taken place throughout the remarkably thick sediment sequences. This process is much more extensive and much more silica is involved than in common chert formation, which results only in thin cherty interbeds or lenses (it should be noted that cherts were not analyzed in the present investigation).

Approximate evaluation of quantitative relations between other compounds in bulk analyses (i.e., composition on a silica-free basis) shows that no essential changes take place when silicification appears. Thus, the process should be interpreted as simple addition of silica, which probably fills pore space, leading to hard rock formation from porous soft sediments.

The source of large amounts of silica needed for this process is still unknown. It does not seem possible to obtain this silica from dissolved siliceous microfossils, because volume problems arise. The dissolution and recrystallization of biogenic opal to form cristobalitic or 
chalcedonic matrix in large amounts in sedimentary sequence would lead to volume reduction, which was not observed. An essential addition of silica to primary sediments is probably necessary.

\section{REFERENCES}

Bischoff, J. L., 1969. Red Sea geothermal Brine deposits-their mineralogy, chemistry and genesis. In Hot brines and recent heavy metal deposits in the Red Sea: New York (Springer-Verlag, Inc.), p. 368.
Boström, K. and Peterson, M. N. A., 1969. The origin of aluminum-poor ferromanganoan sediments in areas of high heat-flow on the East Pacific Rise, Marine Geol., v. 7, p. 427.

Bolstöm, K., Peterson, M. N. A., Joensuu, O., and Fisher, D. E., 1969. Aluminum-poor ferromanganoan sediments on active oceanic ridges, J. Geophys. Res., v. 74, p. 3261.

Skornyakova, N. S., 1976. Dispersed Fe, Mn, Ti and several minor elements in the Pacific sediments. In Bezrukov, P. L. (Ed.), Ferromanganese nodules of the Pacific Ocean, Chapter VI: Moscow Publ. (House “Nauka"), p. 168-181. 